\title{
Review Article \\ The First Moment of Azimuthal Anisotropy in Nuclear Collisions from AGS to LHC Energies
}

\author{
Subhash Singha, Prashanth Shanmuganathan, and Declan Keane
}

Department of Physics, Kent State University, Ohio 44242, USA

Correspondence should be addressed to Subhash Singha; ssingha@kent.edu

Received 22 June 2016; Accepted 29 September 2016

Academic Editor: Emil Bjerrum-Bohr

Copyright ( 2016 Subhash Singha et al. This is an open access article distributed under the Creative Commons Attribution License, which permits unrestricted use, distribution, and reproduction in any medium, provided the original work is properly cited. The publication of this article was funded by SCOAP S $^{3}$

\begin{abstract}
We review topics related to the first moment of azimuthal anisotropy $\left(v_{1}\right)$, commonly known as directed flow, focusing on both charged particles and identified particles from heavy-ion collisions. Beam energies from the highest available, at the CERN LHC, down to projectile kinetic energies per nucleon of a few $\mathrm{GeV}$ per nucleon, as studied in experiments at the Brookhaven AGS, fall within our scope. We focus on experimental measurements and on theoretical work where direct comparisons with experiment have been emphasized. The physics addressed or potentially addressed by this review topic includes the study of Quark Gluon Plasma and, more generally, investigation of the Quantum Chromodynamics phase diagram and the equation of state describing the accessible phases.
\end{abstract}

\section{Introduction}

The purpose of relativistic nuclear collision experiments is the creation and study of nuclear matter at high energy densities. Experiments have established a new form of strongly interacting matter, called Quark Gluon Plasma (QGP) [1-7]. Collective motion of the particles emitted from such collisions is of special interest because it is sensitive to the equation of state in the early stages of the reaction [8-10]. Directed flow was the first type of collective motion identified among the fragments from nuclear collisions [11-13] and, in current analyses, is characterized by the first harmonic coefficient in the Fourier expansion of the azimuthal distribution of the emitted particles with respect to each event's reaction plane azimuth $(\Psi)$ [14-16]:

$$
v_{1}=\langle\cos (\phi-\Psi)\rangle,
$$

where $\phi$ is the azimuth of a charged particle, or more often, the azimuth of a particular particle species, and the angle brackets denote averaging over all such particles in all events. In some experimental analyses, $v_{1}$ is evaluated directly from (1); then a correction is applied for reaction plane resolution [15], whereas, in a typical modern analysis method, the directed flow correlation is extracted using cumulants [16]. In general, $v_{1}$ is of interest when plotted as a function of rapidity, $y$, or sometimes pseudorapidity $\eta=-\ln (\tan \theta / 2)$, where $\theta$ is the polar angle of the particle. The dependence of $v_{1}$ on collision centrality and on transverse momentum, $p_{T}$, can offer additional insights.

Until relatively recently $[17,18]$, the rapidity-even component $v_{1}^{\text {even }}(y)=v_{1}^{\text {even }}(-y)$ was always assumed to be zero or negligible in mass-symmetric collisions. In fact, fluctuations within the initial-state colliding nuclei, unrelated to the reaction plane, can generate a significant $v_{1}^{\text {even }}$ signal $[17,18]$. This fluctuation effect falls beyond the scope of the present review, which focuses on fluid-like directed flow, $v_{1}^{\text {odd }}(y)=-v_{1}^{\text {odd }}(-y)$, as per $(1)$, and from here on, $v_{1}$ for masssymmetric collisions implicitly signifies $v_{1}^{\text {odd }}$.

During the first decade of the study of $v_{1}$ in nuclear collisions, it was more commonly called sideward flow. It refers to a sideward collective motion of the emitted particles and is a repulsive collective deflection in the reaction plane. By convention, the positive direction of $v_{1}$ is taken to be the direction of "bounce-off" of projectile spectators in a fixed target experiment $[8,10]$. Models imply that directed flow, especially the component closest to beam rapidity, is initiated during the passage time of the two colliding nuclei; 
the typical time-scale for this is $2 R / \gamma[9,10]$, where $R$ and $\gamma$ are the nuclear radius and Lorentz factor, respectively. This is even earlier than the still-early time when elliptic flow, $v_{2}$, is mostly imparted. Thus $v_{1}$ can probe the very early stages of the collision $[19,20]$, when the deconfined state of quarks and gluons is expected to dominate the collision dynamics $[9,10]$. Both hydrodynamic $[21,22]$ and transport model $[23,24]$ calculations indicate that the directed flow of charged particles, especially baryons at midrapidity, is sensitive to the equation of state and can be used to explore the QCD phase diagram.

The theoretical work leading to the prediction of collective flow in nuclear collisions evolved gradually. In the mid1950s, Belenkij and Landau [25] were the first to consider a hydrodynamic description of nuclear collisions. During the 1970s, as the Bevatron at Lawrence Berkeley National Lab was converted for use as the first accelerator of relativistic nuclear beams, the idea of hydrodynamic shock compression of nuclear matter emerged [26-28], and these developments in turn led to increasingly realistic predictions [11, 12, 29] that paved the way for the first unambiguous measurement of directed flow at the Bevalac in the mid-1980s [13]. A frequent focus of theory papers during the subsequent years was the effort to use directed flow measurements to infer the incompressibility of the nuclear equation of state in the hadron gas phase and to infer properties of the relevant momentumdependent potential $[8,10]$. The observed directed flow at AGS energies [30-38] and below is close to a linear function of rapidity throughout the populated region, and the slope $d v_{1} / d y$ can adequately quantify the strength of the signal. At SPS energies and above [39-47], a more complex structure is observed in $v_{1}(y)$, with the slope $d v_{1} / d y$ in the midrapidity region being different from the slope in the regions closer to beam rapidity.

Various models also exhibit this kind of behavior. At these energies, both hydrodynamic and nuclear transport calculations predict a negative sign for charged particle $d v_{1} / d y$ near midrapidity, where pions are the dominant particle species. This negative $d v_{1} / d y$ near midrapidity has been given various names in the literature: "third flow component" [48], "antiflow" [49], or "wiggle" [10, 22, 50]. This phenomenon has been discussed as a possible QGP signature, and a negative $d v_{1} / d y$ for baryons has been argued [22] to be particularly significant. However, some aspects of antiflow can be explained in a model with only hadronic physics $[50,51]$ by assuming either incomplete baryon stopping with a positive space-momentum correlation [50], or full stopping with a tilted source [52].

A three-fluid hydrodynamic model [22] predicts a monotonic trend in net-baryon directed flow versus beam energy in the case of a purely hadronic equation of state, whereas a prominent minimum at AGS energies, dubbed the "softest point collapse," is predicted when the equation of state incorporates a first-order phase transition between hadronic and quark-gluonic matter. Recent measurements of both proton and net-proton directed flow at RHIC [47] indeed indicate nonmonotonic directed flow as a function of beam energy, with the minimum lying between 11.5 and $19.6 \mathrm{GeV}$ in $\sqrt{s_{\mathrm{NN}}}$. However, more recent hydrodynamic and nuclear transport calculations which incorporate significant theoretical improvements (see Section 5) do not reproduce the notable qualitative features of the data and therefore cast doubt on any overall conclusion about the inferred properties of the QCD phase diagram. Directed flow has also been measured at the LHC [53]. A negative slope of $v_{1}(\eta)$ is observed for charged particles, but its magnitude is much smaller than at RHIC, which is thought to be a consequence of the smaller tilt of the participant zone at the LHC.

In this article, we review a representative set of directed flow results spanning AGS to LHC energies. In Sections 2 and 3, we discuss measurements of $v_{1}$ for charged particles in mass-symmetric and mass-asymmetric collisions, respectively. In Section 4, we cover measurements of $v_{1}$ for various identified particle species. Section 5 reviews some recent model calculations which lend themselves to direct comparisons with directed flow data. Section 6 presents a summary and future outlook.

\section{Differential Measurements of Charged Particle Directed Flow}

In this section, we review measurements of $v_{1}$ for all charged particles in cases where individual species were not identified. Studies of the dependence on transverse momentum $p_{T}$, pseudorapidity $\eta$, beam energy $\sqrt{s_{\mathrm{NN}}}$, system size, and centrality are included.

2.1. Dependence of $v_{1}$ on Transverse Momentum. The $p_{T^{-}}$ dependence of $v_{1}$ for charged particles has been studied by the STAR experiment at RHIC [43, 44]. Figure 1(a) presents directed flow results for $\mathrm{Au}+\mathrm{Au}$ collisions in two centrality intervals, $5-40 \%$ and $40-80 \%$, and in two regions of pseudorapidity, $|\eta|<1.3$ and $2.5<|\eta|<1.3$. In this case, because of the odd-functional property of $v_{1}(\eta)$, the backward pseudorapidity region by convention has its sign of $v_{1}$ reversed before summing over the indicated gate in pseudorapidity. These measurements represent the first instance of using only spectators to determine the estimated azimuth of the reaction plane [54-56].

The measured $v_{1}$ in the midpseudorapidity interval $|\eta|<$ 1.3 crosses zero in the $p_{T}$ region of 1 to $2 \mathrm{GeV} / c$. Extrapolations raise the possibility that a qualitatively similar zero crossing by forward pseudorapidity $v_{1}$ occurs at higher $p_{T}$, but that region does not fall within the acceptance of the STAR detector.

The charged particle $v_{1}\left(p_{T}\right)$ measurements by ALICE [53] in $\mathrm{Pb}+\mathrm{Pb}$ collisions at $2.76 \mathrm{TeV}$ are compared in Figure 1(b) with the corresponding data from $200 \mathrm{GeV} \mathrm{Au}+\mathrm{Au}$ collisions at RHIC. The measurements at both LHC and RHIC show a similar trend, including a sign change around $p_{T} \sim 1.5 \mathrm{GeV} / c$ in central collisions and negative values at all $p_{T}$ for peripheral collisions.

There is an interest in the observation of zero crossing behavior in $v_{1}\left(p_{T}\right)$ as it can be used to constrain hydrodynamic model calculations [57]. It has been pointed out that this sign change is an artifact of combining all species of charged particles together and can be explained [44] by the 


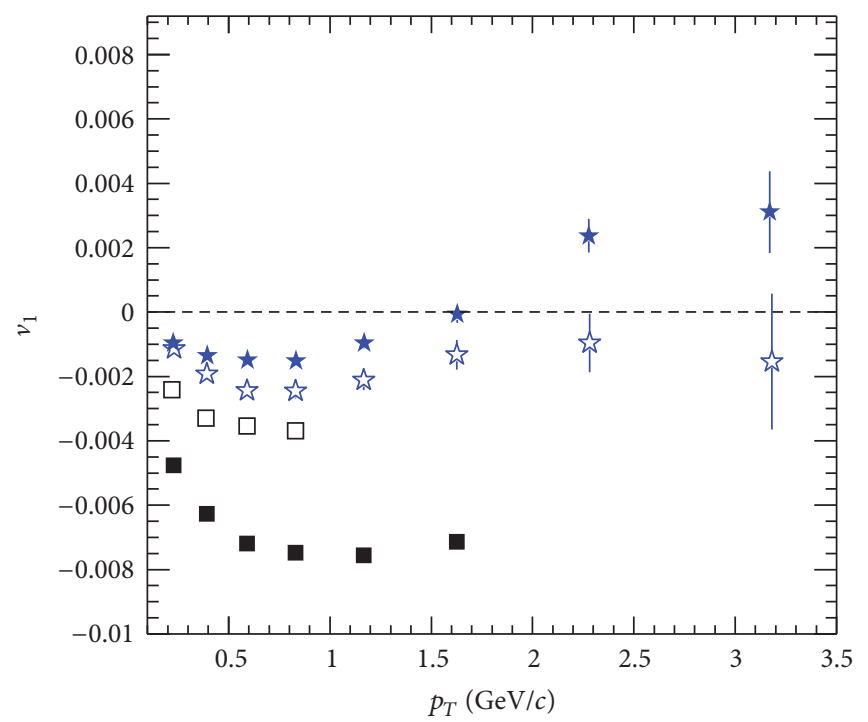

$\mathrm{Au}+\mathrm{Au} 200 \mathrm{GeV}$

$\begin{array}{ll}|\eta|<1.3 & 2.5<\eta<4 \\ \star 5-40 \% & \square 5-40 \% \\ \text { 矢 } 40-80 \% & \square 40-80 \%\end{array}$

(a)

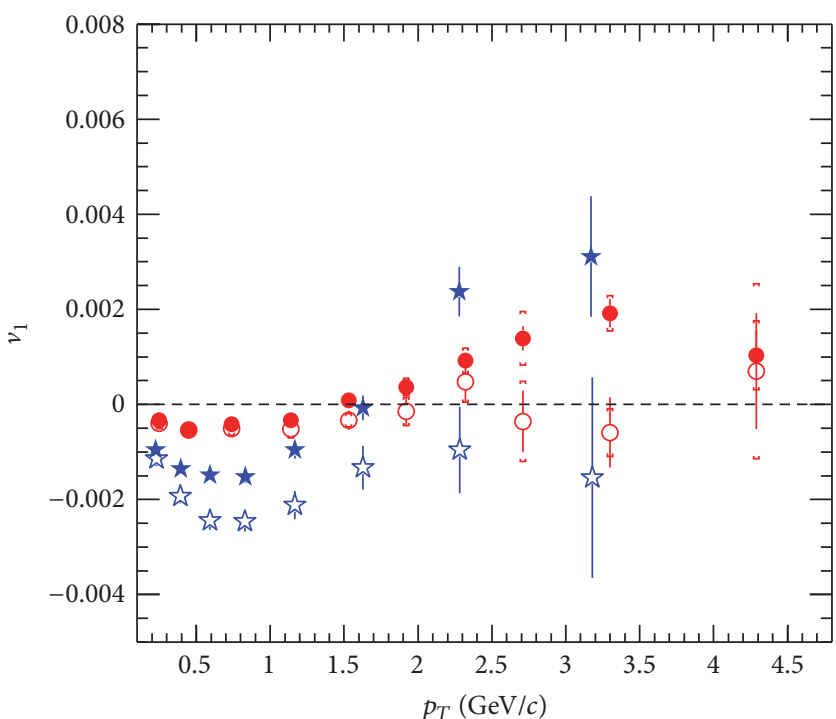

$\mathrm{Pb}+\mathrm{Pb} 2.76 \mathrm{TeV}$

- $5-40 \%$

○ $40-80 \%$

$\mathrm{Au}+\mathrm{Au} 200 \mathrm{GeV}$

$\star 5-40 \%$

is $40-80 \%$

(b)

FIgURE 1: (a) Charged particle $v_{1}$ as a function of transverse momentum in $200 \mathrm{GeV} \mathrm{Au+Au} \mathrm{collisions} \mathrm{at} \mathrm{RHIC,} \mathrm{for} \mathrm{two} \mathrm{centralities} \mathrm{and} \mathrm{two}$ pseudorapidity windows $(|\eta|<1.3$ and $2.5<\eta<1.3)$ [44]. (b) Comparison of RHIC results with midrapidity measurements in $2.76 \mathrm{TeV}$ $\mathrm{Pb}+\mathrm{Pb}$ collisions at the LHC [53].

different sign of $v_{1}$ for pions and baryons, in conjunction with the enhanced production of baryons at higher $p_{T}$ [58]. This complication is one of the reasons why directed flow of identified particles, as reviewed in later sections, can be easier to interpret and offers additional insights.

2.2. Dependence of $v_{1}$ on Pseudorapidity. Figure 2(a) shows charged particle $v_{1}(\eta)$ in $\mathrm{Au}+\mathrm{Au}$ collisions at 19.6, 62.4, 130, and $200 \mathrm{GeV}$ measured in the PHOBOS detector [42]. It is evident that charged particle $v_{1}$ within 3 to 4 units of $\eta$ on either side of $\eta=0$ has a sign opposite to that of the spectators on that side of $\eta=0$ (the antiflow phenomenon).

Figure 2(b) shows the $\eta$ dependence of $v_{1}$ for charged particles in $2.76 \mathrm{TeV} \mathrm{Pb}+\mathrm{Pb}$ collisions, as measured by the ALICE collaboration [53] at the LHC. The ALICE results are compared in this panel with RHIC measurements from STAR $[41,44]$. The $v_{1}$ slope at the LHC and at the top RHIC energies has the same negative sign, but the slope magnitude at the LHC is a factor $\sim 3$ smaller than at top RHIC energy. This pattern is consistent with the participant zone at the LHC having a smaller tilt, as predicted [52], and does not support a proposed picture at $\mathrm{LHC}$ energies in which a strong rotation is imparted to the central fireball $[59,60]$.

2.3. System Size and Beam Energy Dependence of $v_{1}$. The beam energy and system size dependence of $v_{1}$ have been studied at RHIC using data from two colliding species at two beam energies. Figure 3(a) shows charged particle $v_{1}$ for midcentrality (30-60\%) $\mathrm{Au}+\mathrm{Au}$ and $\mathrm{Cu}+\mathrm{Cu}$ collisions at
$\sqrt{s_{\mathrm{NN}}}=62.4$ and $200 \mathrm{GeV}$, measured by STAR [44]. A trend of decreasing $v_{1}(\eta)$ is observed as beam energy increases for both $\mathrm{Au}+\mathrm{Au}$ and $\mathrm{Cu}+\mathrm{Cu}$ collisions. Across the reported pseudorapidity range, $v_{1}(\eta)$ is independent of system size within errors at each beam energy. This is a remarkable finding, given that the $\mathrm{Au}+\mathrm{Au}$ system mass is three times that of the $\mathrm{Cu}+\mathrm{Cu}$ system, and given that neither the AMPT [61$63]$ nor the UrQMD $[23,24]$ models exhibit such a scaling behavior.

A different scaling behavior is presented in Figure 3(b). Here, the data in Figure 3(a) are transformed into the rest frame of the beam nucleus; that is, zero on the $x$-axis corresponds to $y_{\text {beam }}$ for each of the two collision energies involved. Within errors, the measurements lie on a universal curve across about three units of pseudorapidity. This behavior is known in the heavy-ion literature as limiting fragmentation, and had previously been observed in $\mathrm{Au}+\mathrm{Au}$ collisions as a function of beam energy by STAR [43] and PHOBOS [42]. The term "limiting fragmentation" was originally employed by Feynman [64] and Benecke et al. [65] to describe the analogous phenomenon of the measured $\mu^{+} / \mu^{-}$ratio in cosmic ray showers at sea level being almost independent of muon energy.

2.4. Centrality Dependence of $v_{1}$. Figure 4 shows $v_{1}$ as a function of collision centrality in 62.4 and $200 \mathrm{GeV} \mathrm{Au}+\mathrm{Au}$ [44] from STAR and in $2.76 \mathrm{TeV} \mathrm{Pb}+\mathrm{Pb}$ [53] from ALICE, for the midpseudorapidity region $|\eta|<1$.3. Figure 4 also shows $v_{1}$ (divided by 6 in order to fit conveniently on a common scale) as a function of collision centrality in $200 \mathrm{GeV} \mathrm{Au}+\mathrm{Au}$ 


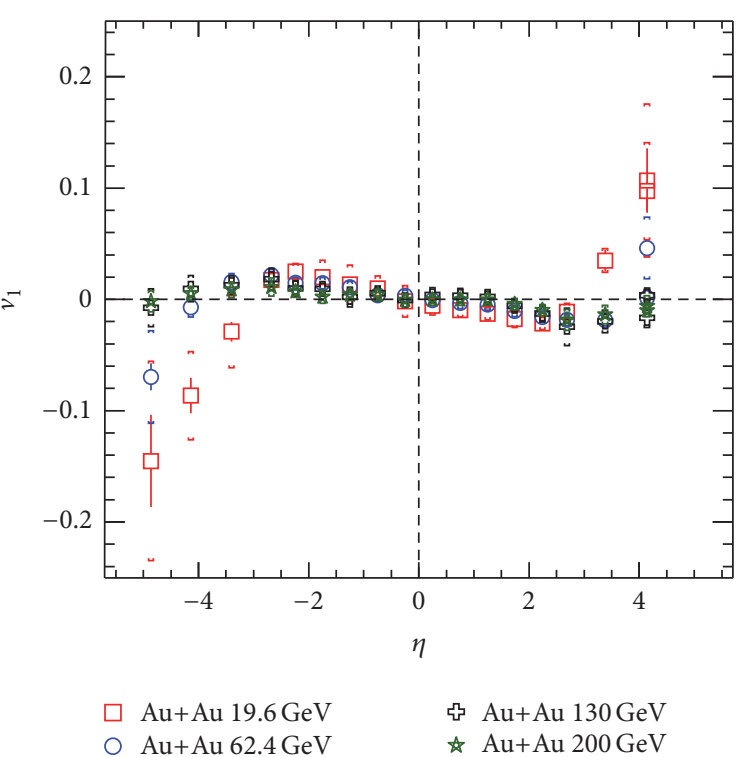

(a)

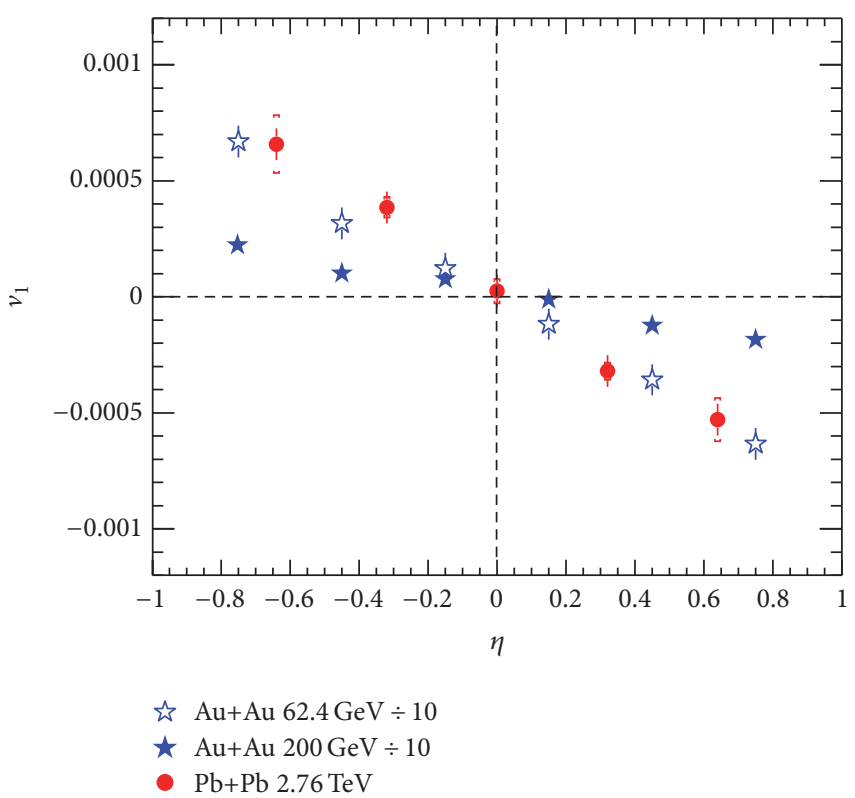

(b)

FIGURE 2: (a) Charged particle $v_{1}$ as a function of $\eta$ for $0-40 \%$ central Au+Au collisions at $\sqrt{s_{\mathrm{NN}}}=19.6,62.4,130$, and $200 \mathrm{GeV}$, measured by PHOBOS at RHIC [42]. (b) Comparison of charged particle $v_{1}$ versus $\eta$ at RHIC [44] and LHC [53] energies. Note that all the RHIC data points are divided by 10 in order to be plotted on a common scale with LHC results.

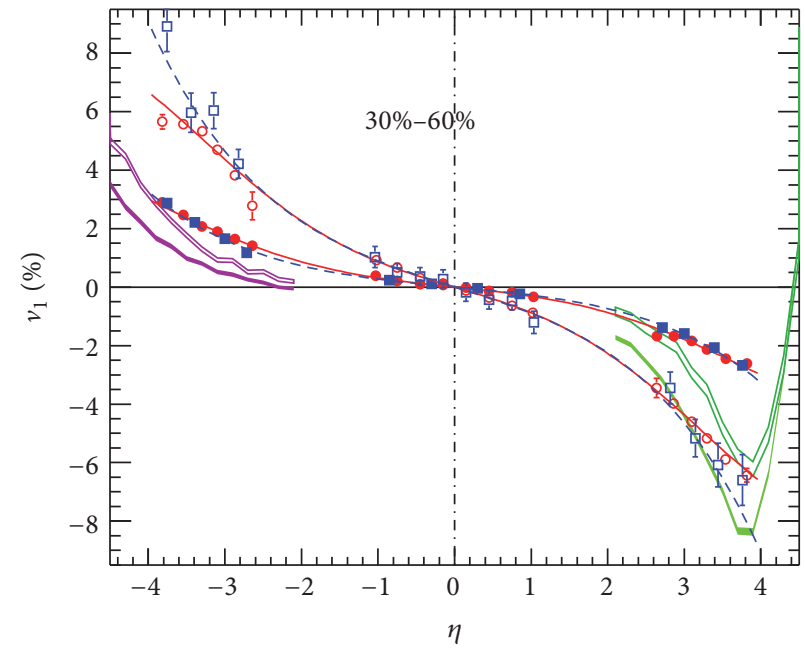

Data

$\begin{array}{ll}\text { - } & 200 \mathrm{GeV} \mathrm{Au}+\mathrm{Au} \\ \text { - } & 200 \mathrm{GeV} \mathrm{Cu}+\mathrm{Cu} \\ \text { - } & 62.4 \mathrm{GeV} \mathrm{Au}+\mathrm{Au} \\ \text { - } & 62.4 \mathrm{GeV} \mathrm{Cu}+\mathrm{Cu}\end{array}$

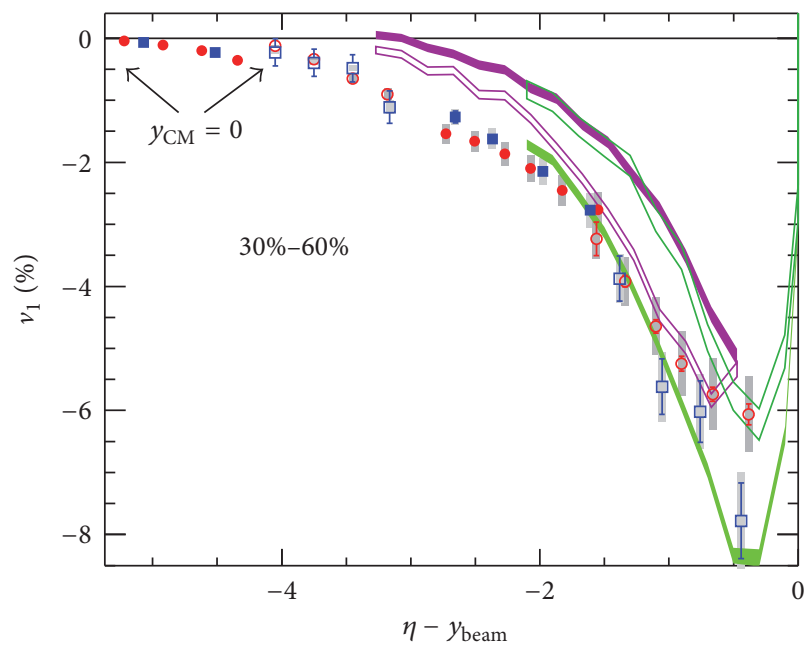

Data

- $200 \mathrm{GeV} \mathrm{Au}+\mathrm{Au}$

- $200 \mathrm{GeV} \mathrm{Cu}+\mathrm{Cu}$

- $62.4 \mathrm{GeV} \mathrm{Au}+\mathrm{Au}$

ㅁ $62.4 \mathrm{GeV} \mathrm{Cu}+\mathrm{Cu}$
AMPT

$=200 \mathrm{GeV} \mathrm{Au}+\mathrm{Au}$

$-200 \mathrm{GeV} \mathrm{Cu}+\mathrm{Cu}$

$-62.4 \mathrm{GeV} \mathrm{Au}+\mathrm{Au}$

$=62.4 \mathrm{GeV} \mathrm{Cu}+\mathrm{Cu}$

(a)

(b)

Figure 3: (a) Charged particle $v_{1}$ as a function of $\eta$ for 200 and $62.4 \mathrm{GeV} \mathrm{Au}+\mathrm{Au}$ and $\mathrm{Cu}+\mathrm{Cu}$ collisions [44]. (b) is the same as (a), but with the $x$-axis shifted by $y_{\text {beam }}$.

[44] from STAR at the forward pseudorapidity region $2.5<$ $|\eta|<4.0$.

Directed flow magnitude at midpseudorapidity increases monotonically going from central to peripheral collisions at all three beam energies, and there is a strong trend for this magnitude to decrease with increasing beam energy. A similar but stronger centrality dependence is observed at forward pseudorapidity, and the magnitude also increases strongly from midpseudorapidity to forward pseudorapidity.

It has been pointed out by Caines [67] that many aspects of soft physics (defined as $p_{T}<2 \mathrm{GeV} / c$ ) in heavy-ion collisions at relativistic energies depend only on event multiplicity 


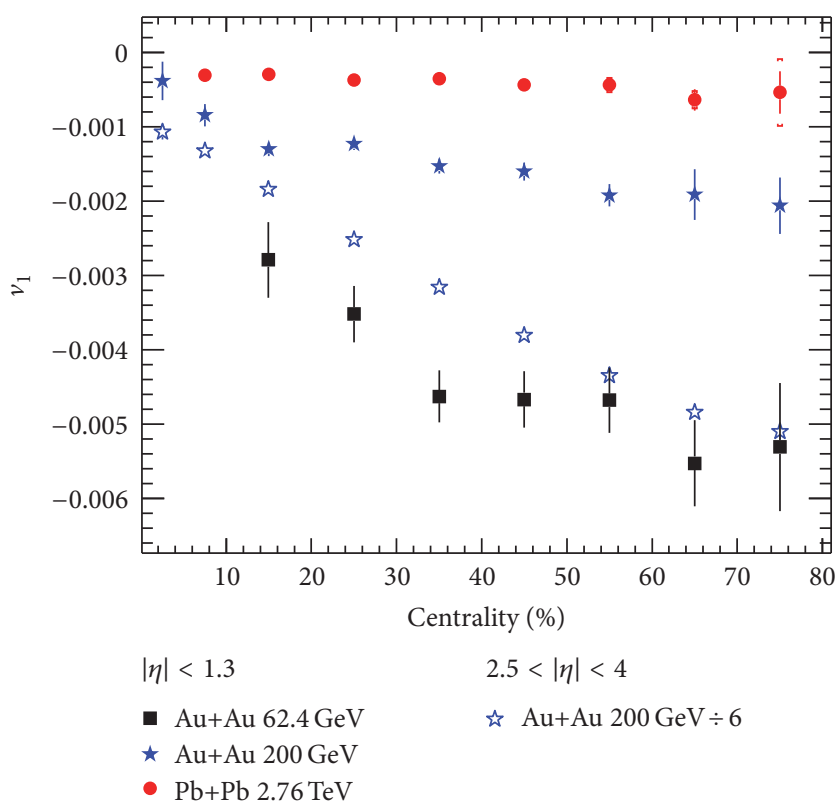

FIGURE 4: $v_{1}$ of charged particles as a function of centrality for a midpseudorapidity region $(|\eta|<1.3)$ and a forward pseudorapidity region $(2.5<|\eta|<4.0)$. Two RHIC datasets, 62.4 and $200 \mathrm{GeV}$ $\mathrm{Au}+\mathrm{Au}[44]$, and one LHC dataset, $2.76 \mathrm{TeV} \mathrm{Pb}+\mathrm{Pb}$ [53], are shown at midpseudorapidity, while only $200 \mathrm{GeV} \mathrm{Au+Au} \mathrm{[44]} \mathrm{is} \mathrm{shown}$ at forward pseudorapidity. Note that the data points at forward pseudorapidity are divided by 6 in order to be plotted on a common scale with the data at midpseudorapidity.

per unit rapidity; in other words, for a fixed value of $d N_{\mathrm{ch}} / d \eta$, there is no significant dependence on beam energy, or on centrality, or on the mass of the colliding system. This type of scaling is called "entropy-driven" soft physics. The directed flow results for two beam energies and two colliding systems reported by STAR in [44] (see Figure 3) represented one of the first (and still few) violations of entropy-driven multiplicity scaling. In contrast, this scaling is observed to hold, with caveats, for homogeneity lengths from femtoscopy $[67,68]$, for elliptic flow per average participant eccentricity $[67,69]$, and for various strangeness yields [67].

\section{Directed Flow of Charged Particles in Mass- Asymmetric Collisions}

In mass-asymmetric collisions like $\mathrm{Cu}+\mathrm{Au}$, the well-defined distinction that exists in mass-symmetric collisions between the odd $v_{1}(\eta)$ component (a hydrodynamic effect correlated with the reaction plane) and the even $v_{1}(\eta)$ component (an initial-state fluctuation effect unrelated to the reaction plane) no longer holds. In a recent paper from the PHENIX collaboration, they report midrapidity charged hadron $v_{1}\left(p_{T}\right)$ in $\mathrm{Cu}+\mathrm{Au}$ collisions at $\sqrt{s_{\mathrm{NN}}}=200 \mathrm{GeV}$ for centralities of $10-20 \%, 20-30 \%, 30-40 \%$, and $40-50 \%$, using spectator neutrons from the $\mathrm{Au}$ side of the collision to determine the event plane; see Figure 5 [66]. However, they preserve the standard convention for the sign of $v_{1}$ by defining the direction of bounce-off by remnants of the first nucleus in the
$\mathrm{A}+\mathrm{A}$ system $(\mathrm{Cu})$ to be positive. An even more recent paper from STAR reports $v_{1}\left(p_{T}\right)$ distributions for the same system and centrality that are consistent within errors [70].

The PHENIX results in Figure 5 [66] reveal that the higher $p_{T}$ particles at midrapidity (above 1 or $1.5 \mathrm{GeV} / c$ in $\left.p_{T}\right)$ and at all the studied centralities have negative $v_{1}$ and so are preferentially emitted with azimuths parallel to the $\mathrm{Au}$ fragment bounce-off direction (and antiparallel to the $\mathrm{Cu}$ fragment bounce-off direction). Whether or not the more abundant particles below $1 \mathrm{GeV} / c$ are preferentially emitted with opposite azimuths, as might be expected based on momentum conservation, cannot be answered within the systematic uncertainty of the measurements [66].

In the STAR collaboration's analysis of charged particle directed flow in $\mathrm{Cu}+\mathrm{Au}$ collisions at $\sqrt{\boldsymbol{s}_{\mathrm{NN}}}=200 \mathrm{GeV}$, a particular focus is the $v_{1}$ difference between positive and negative charges. This difference has the potential to be sensitive to the strong electric field between the two incident ions, whose electric charges differ by $79-29=50$ units; this field has a lifetime on the order of a fraction of a $\mathrm{fm} / c$. Figure 6 [70] presents $v_{1}(\eta)$ at medium $p_{T}\left(1<p_{T}<2 \mathrm{GeV} / c\right)$ and intermediate centrality (10-40\%). Like the PHENIX result, this $v_{1}$ measurement was made relative to the event plane from spectator neutrons, dominated by the $\mathrm{Au}$ side. It is evident from Figure 6 that both even and odd components are present, and most interestingly, there is a significant pattern showing a larger magnitude for negative particles.

The Parton-Hadron String Dynamics (PHSD) model [72, 73], when the initial electric field is explicitly modeled, predicts a $v_{1}$ difference signal that is an order of magnitude larger than observed [70]. On the other hand, parton distribution functions [74] can be used to estimate the number of quarks and antiquarks at very early times in relation to the number created in the collision; then given certain plausible assumptions, as set out in [70], it can be inferred that only a small fraction of the total quarks created in the collision are produced during the lifetime of the initial electric field. In addition to this important insight, the charged-dependent directed flow measurements in $\mathrm{Cu}+\mathrm{Au}$ collisions offer new and valuable quantitative information with relevance to the Chiral Magnetic Effect $[75,76]$ and the Chiral Magnetic Wave $[77,78]$.

\section{Differential Measurements of Identified Particle Directed Flow}

The charged particle measurements reviewed in Section 2 are an admixture of all emitted particle species. Measurements of directed flow for identified particles offer more insights into the underlying physics that controls this observable. In this section, we discuss the dependence of $v_{1}$ on $p_{T}, y$, and centrality for several identified particle species.

4.1. Dependence of $v_{1}$ on Transverse Momentum. Measurements of $v_{1}\left(p_{T}\right)$ for protons, antiprotons, and charged pions have been reported by the E877 collaboration at the AGS $(11 \mathrm{~A} \mathrm{GeV/c})$ [30-33]. For antiprotons, large negative values of $v_{1}$ are observed for $p_{T}>0.1 \mathrm{GeV} / c$ but with large statistical 


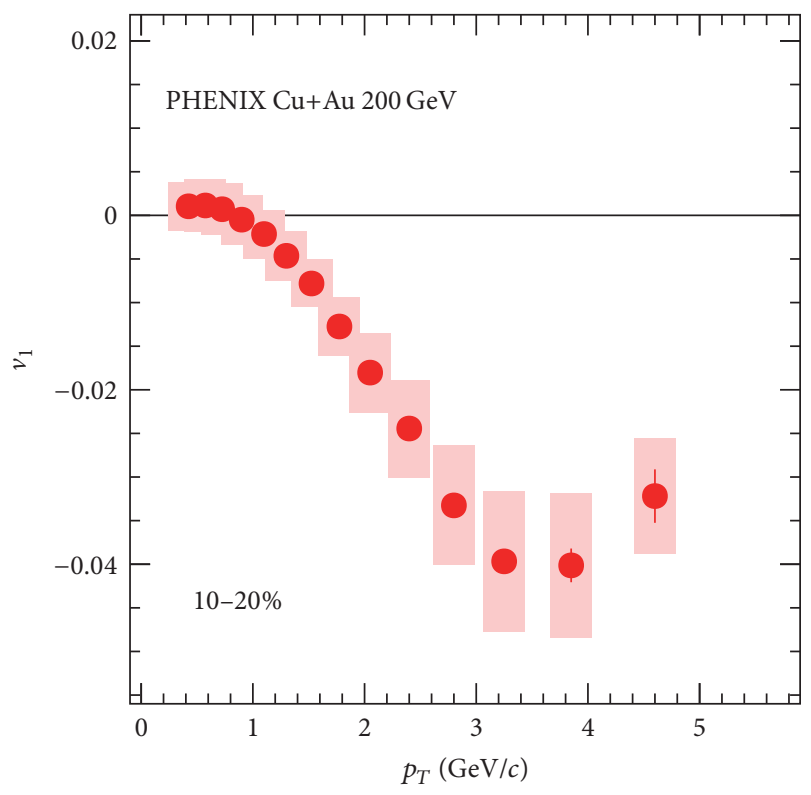

$h^{ \pm}$

(a)

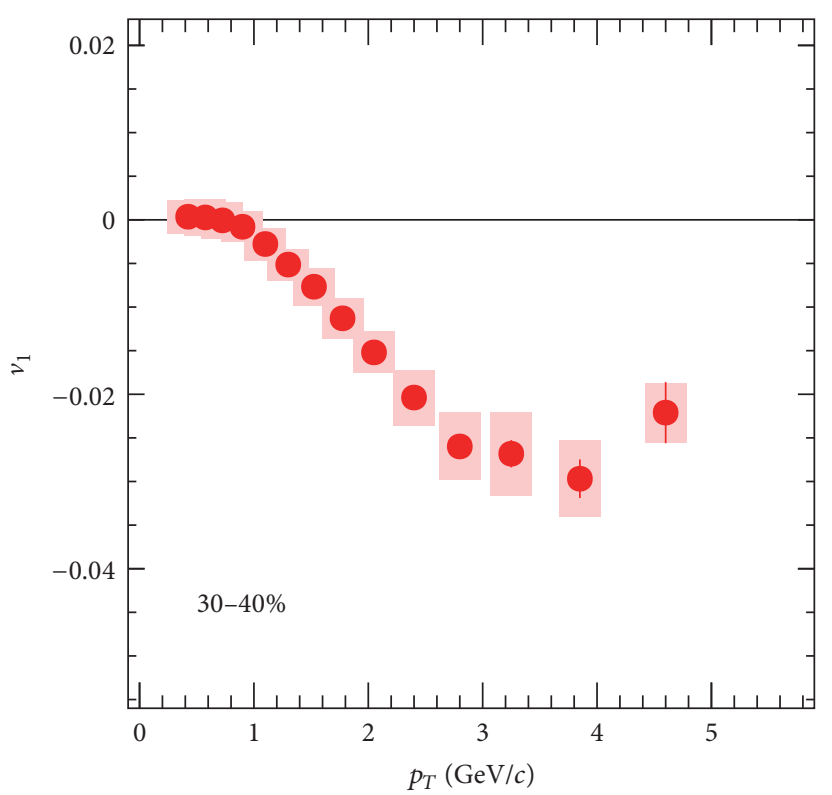

$h^{ \pm}$

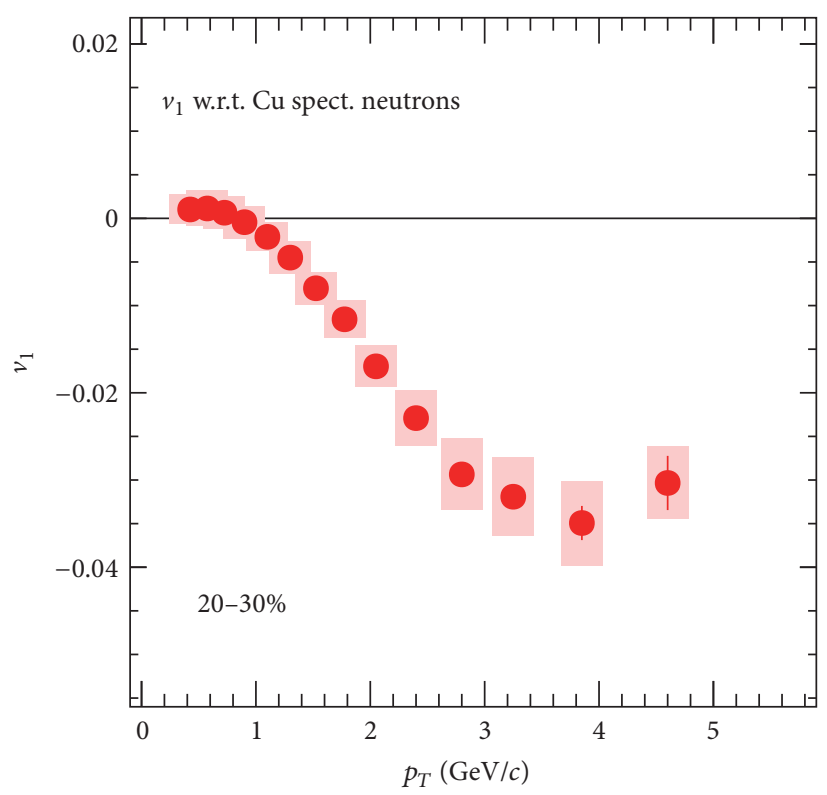

$h^{ \pm}$

(b)

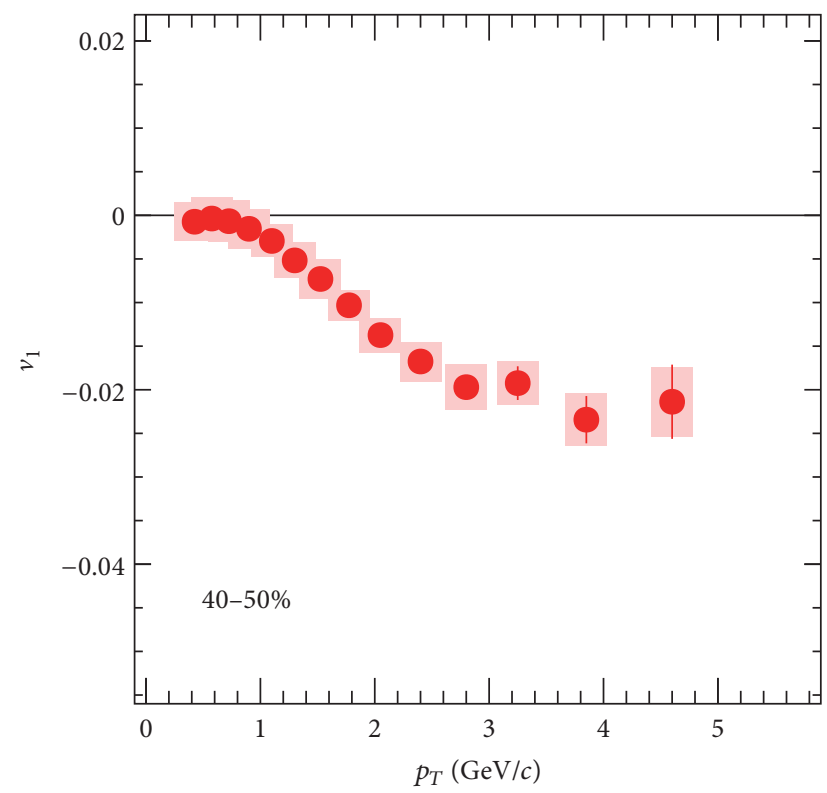

$h^{ \pm}$

(c)

(d)

Figure 5: Midrapidity $v_{1}\left(p_{T}\right)$ in $\mathrm{Cu}+\mathrm{Au}$ collisions at $\sqrt{s_{\mathrm{NN}}}=200 \mathrm{GeV}$ in four bins of centrality, as reported by the PHENIX collaboration [66].

errors. For protons and charged pions, $v_{1}\left(p_{T}\right)$ in various rapidity gates have been published, and these results are also divided into various bins of transverse energy, $E_{T}$, which is a proxy for centrality. The E877 collaboration also provides the information needed to convert from their intervals of $E_{T}$ into percent centrality [31].
The NA49 collaboration [39] measured proton and pion $v_{1}\left(p_{T}\right)$ in $\mathrm{Pb}+\mathrm{Pb}$ collisions at a projectile kinetic energy of $158 \mathrm{~A} \mathrm{GeV}$, as shown in Figure 7. The rapidity gate for these measurements is $4<y_{\text {lab }}<5$, which corresponds to a forward region (midrapidity is $y_{\text {lab }}=2.92$ ). The NA49 collaboration describes the $v_{1}\left(p_{T}\right)$ behavior as "peculiar," 


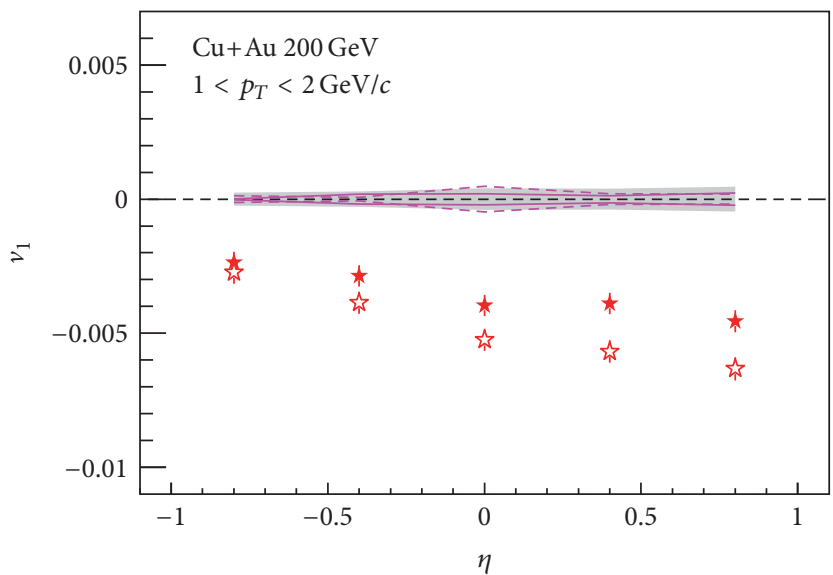

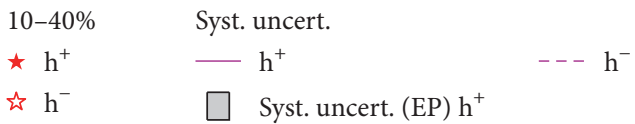

(a)

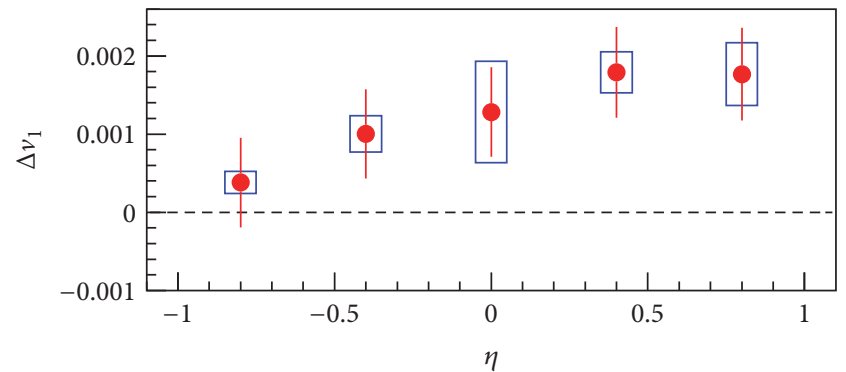

(b)

Figure 6: Directed flow as a function of pseudorapidity, separately evaluated for positively and negatively charged particles, at $1<p_{T}<$ $2 \mathrm{GeV} / c$ and centrality $10-40 \%$ in $200 \mathrm{GeV} \mathrm{Cu}+\mathrm{Au}$ collisions in the STAR detector [70].

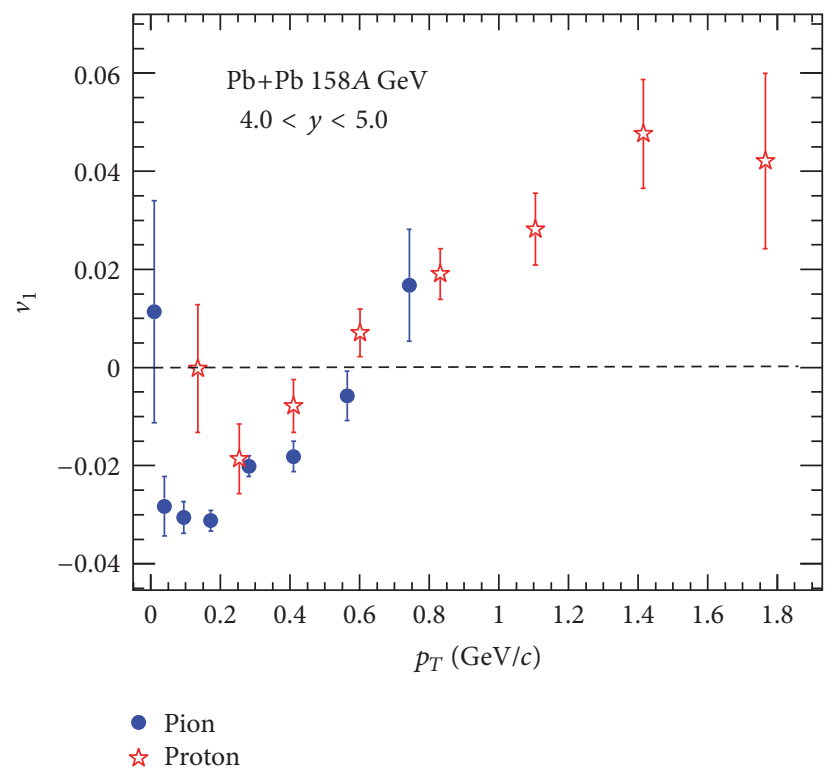

Figure 7: Pion and proton $v_{1}\left(p_{T}\right)$ at $4<y_{\mathrm{lab}}<5$ in $\mathrm{Pb}+\mathrm{Pb}$ collisions at the SPS, at a projectile kinetic energy of $158 \mathrm{~A} \mathrm{GeV} \mathrm{[39].}$

especially for pions. However, they point out that negative $v_{1}$ at low $p_{T}$ has been predicted by Voloshin [79] and is explained by the interaction of radial and directed flow. Various types of nonflow effect were also mentioned as possible contributors to the observed pion behavior [39].

4.2. Dependence of $v_{1}$ on Rapidity. Various models suggest that the structure of $v_{1}(y)$ near midrapidity, especially the pattern for baryons, is sensitive to the QCD equation of state and therefore this signal can be used to investigate QGP production and changes of phase [8-10, 22, 80, 81]. Figure 8 presents proton and pion $v_{1}(y)$ in central, midcentral, and peripheral $\mathrm{Pb}+\mathrm{Pb}$ collisions at projectile kinetic energies of $40 A$ and $158 A \mathrm{GeV}[40]$, as reported by the NA49 collaboration at the CERN SPS. The data points at negative rapidity are mirrored from the positive side. The $v_{1}(y)$ for pions is similar in magnitude and shape at both $40 \mathrm{~A}$ and $158 \mathrm{~A} \mathrm{GeV}$. The proton $v_{1}(y)$ measurements suggest that proton antiflow (also called wiggle) [10, 22, 48-50], which is not observed at the AGS (see Figure 9), might begin to happen at SPS energies. However, based on detailed studies using a variety of methods, including the approach introduced by Borghini et al. [82], the NA49 collaboration reports that the observed pattern of proton $v_{1}(y)$ at SPS energies could be influenced by nonflow effects [40].

The EOS-E895 experiment carried out a beam energy scan at the Brookhaven AGS during 1996. E895 featured the first Time Projection Chamber with pad readout and reported directed flow in the form of $v_{1}$, as well as in the form of the older observable $\left\langle p_{x}\right\rangle$ [83] (in-plane transverse momentum) for several identified particle species: $p, \bar{p}, \Lambda$, $K_{S}^{0}$, and $K^{ \pm}$, in $\mathrm{Au}+\mathrm{Au}$ collisions at projectile kinetic energies $2 A, 4 A, 6 A$, and $8 A \mathrm{GeV}$ [34-38]. Figure 9(a) shows $v_{1}\left(y^{\prime}\right)$ for protons at the four E895 beam energies, where $y^{\prime}$ denotes normalized rapidity such that the target and projectile are always at $y^{\prime}=-1$ and +1 , respectively. The slope of $v_{1}$ remains positive for all four beam energies.

The slope of the rapidity dependence of directed flow was extracted by E895 using a cubic fit $v_{1}\left(y^{\prime}\right)=F y^{\prime}+C y^{\prime 3}$. Figure 9(b) shows the fitted proton slope $d v_{1} / d y$ (not using normalized rapidity) and, unlike in [34], the horizontal axis in Figure 9(b) uses the now-conventional beam energy scale $\sqrt{s_{\mathrm{NN}}}$. Two additional points for proton $d v_{1} / d y$ in $\mathrm{Au}+\mathrm{Au}$ collisions are also plotted here: a measurement at the top 

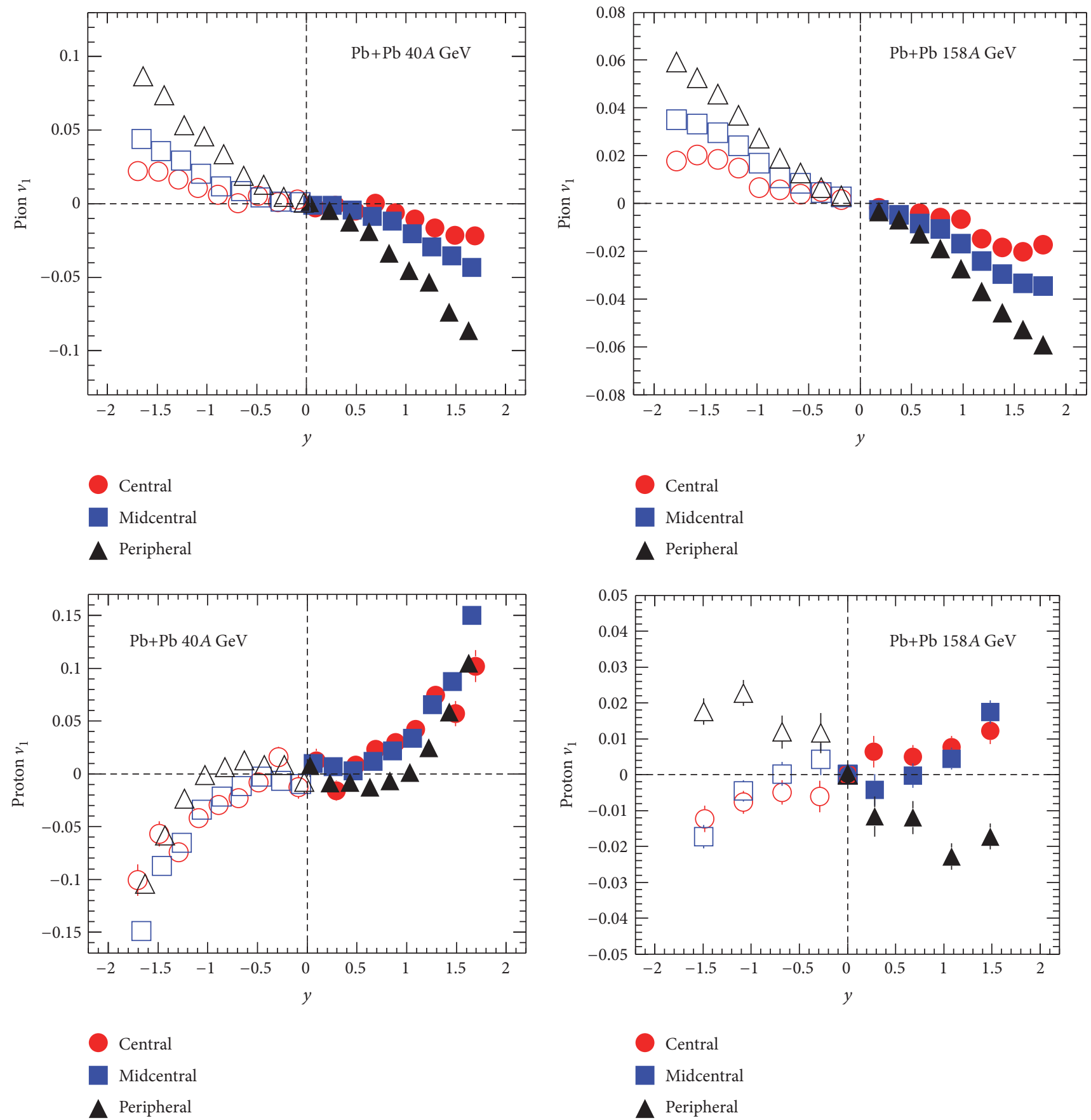

FIGURE 8: Pion and proton $v_{1}(y)$ for three centralities in $40 A$ and $158 \mathrm{~A} \mathrm{GeV} \mathrm{Pb}+\mathrm{Pb}$ collisions at the SPS [40]. The open markers at negative rapidity were obtained by reflecting the solid markers at positive rapidity, where the detector acceptance was optimum.

energy of the Berkeley Bevalac (1.2A GeV) [71] using the same EOS TPC detector as in E895 and an E877 measurement at the top AGS energy of $11 A \mathrm{GeV}$ [31]. The plotted data show a peak near $2 A \mathrm{GeV}$ beam kinetic energy $\left(\sqrt{s_{\mathrm{NN}}} \sim 2.7 \mathrm{GeV}\right)$ and, thereafter, a smooth decrease with beam energy across the AGS range.

Three-fluid hydrodynamic calculations [22] predict a "softest point" in the Equation of State in the AGS energy range, but the E895 beam energy scan did not reveal any nonmonotonic behavior in the energy dependence. Overall, hadronic models with a momentum-dependent mean field [84] show better agreement with data at AGS and SPS energies.

Phase-I of the beam energy scan (BES) $[85,86]$ at RHIC took data in 2010, 2011, and 2014, spanning the $\sqrt{s_{\mathrm{NN}}}$ range from $200 \mathrm{GeV}$ down to $7.7 \mathrm{GeV}$. Lattice QCD calculations [87-89] imply a smooth crossover from hadronic matter to QGP at beam energies near and above the top energy 


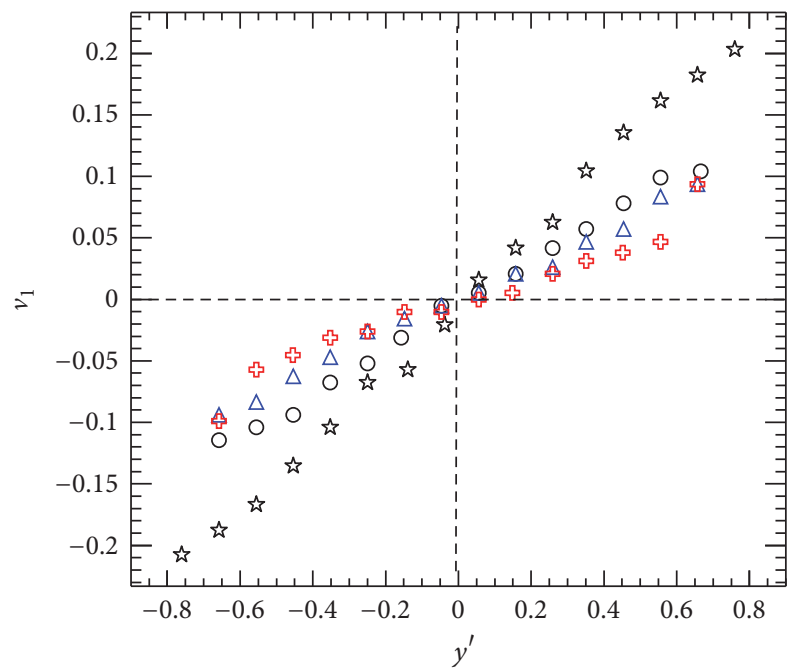

$\mathrm{E} 895 \mathrm{Au}+\mathrm{Au}$

¿ $2 A \mathrm{GeV}\left(0.1<p_{T}<2 \mathrm{GeV} / c\right)$

○ $4 A \mathrm{GeV}\left(0.1<p_{T}<2 \mathrm{GeV} / c\right)$

$\triangle 6 A \mathrm{GeV}\left(0.2<p_{T}<2 \mathrm{GeV} / c\right)$

њ $8 A \mathrm{GeV}\left(0.4<p_{T}<2 \mathrm{GeV} / c\right)$

(a)

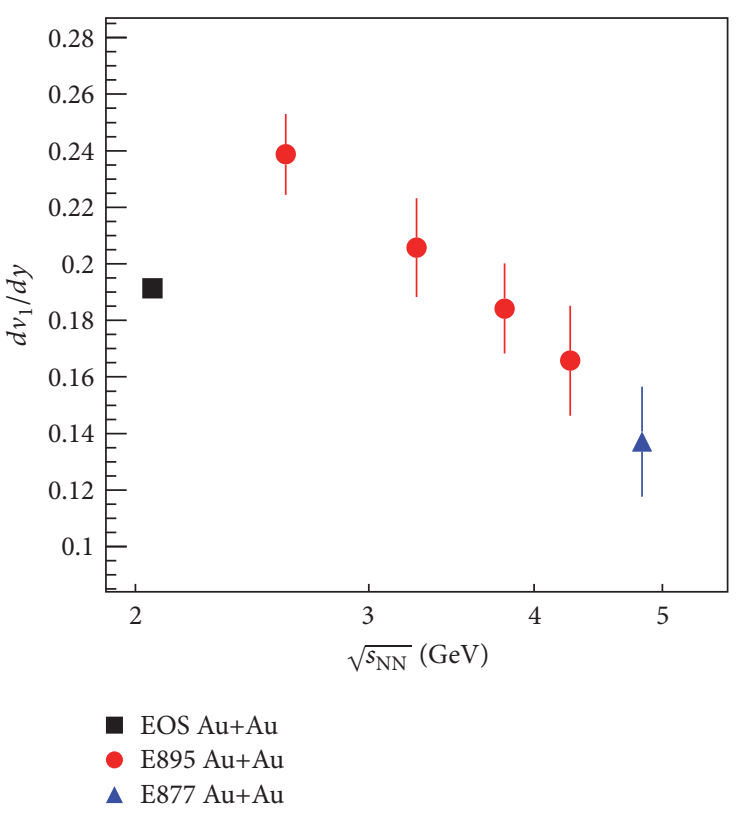

(b)

Figure 9: (a) $v_{1}\left(y^{\prime}\right)$ for protons in 2A, 4A, 6A, and $8 A \mathrm{GeV}$ Au+Au collisions measured by E895 [34]. (b) Beam energy dependence of the slope $d v_{1} / d y$ (here using unnormalized rapidity) for protons, replotted using the more ubiquitous beam energy scale $\sqrt{s_{\mathrm{NN}}}$ [34]. A point from the same detector at the Bevalac [71] and another from E877 at the top AGS energy [31] are also plotted.

of RHIC, whereas phase diagram features like a first-order phase transition and a critical point may become evident in nuclear collisions as the beam energy is scanned across RHIC's BES region. The STAR experiment initially reported measurements of directed flow for protons, antiprotons, and charged pions in the energy range of 7.7 to $200 \mathrm{GeV}$ [47]. At the 2015 Quark Matter meeting, preliminary directed flow data at BES energies for nine particle species were presented, along with $v_{1}(y)$ slopes for protons, $\Lambda$, and $\pi^{+}$in 9 bins of centrality [90]. Figure 10 shows $v_{1}(y)$ at intermediate centrality (10-40\%) for $p, \Lambda, \bar{p}, \bar{\Lambda}, K^{ \pm}, K_{S}^{0}$, and $\pi^{ \pm}$at 7.7 to $39 \mathrm{GeV}$.

The overall strength of directed flow has been characterized by the slope $d v_{1} / d y$ from a linear fit over the range $-0.8<y<0.8$ [90], and the beam energy dependence of these slopes for the same nine particle species $(p, \Lambda, \bar{p}, \bar{\Lambda}$, $K^{ \pm}, K_{S}^{0}$, and $\pi^{ \pm}$) in $10-40 \%$ centrality $\mathrm{Au}+\mathrm{Au}$ collisions is presented in Figure 11.

The most noteworthy feature of the data is a minimum within the $\sqrt{s_{\mathrm{NN}}}$ range of 10 to $20 \mathrm{GeV}$ in the slope $d v_{1} /\left.d y\right|_{y \sim 0}$ for protons at intermediate centrality. The same quantity for $\Lambda$ hyperons is consistent with the proton result, but the larger statistical errors for $\Lambda$ do not allow an independent determination of a possible minimum in the beam energy dependence for this species. The proton and $\Lambda$ directed flow slope change from positive to negative close to $11.5 \mathrm{GeV}$ and remain negative at all the remaining energies (up to and including $200 \mathrm{GeV}$ in the case of protons). The remaining species have negative slope at all the studied beam energies.
At $7.7 \mathrm{GeV}, d v_{1} / d y$ for $K^{-}$is closer to zero than $d v_{1} / d y$ for $K^{+}$, which supports the inference that $K^{+}$and $K^{-}$ experience nuclear potentials that are repulsive and attractive, respectively, under the conditions created at this beam energy [91].

4.3. Net-Particle Directed Flow. There are two separate contributions to the energy dependence of proton directed flow in the vicinity of midrapidity: one part arises from baryon number transported from the initial-state at beam rapidity towards $y \sim 0$ by the stopping process of the collision, while the other part arises from baryon-antibaryon pairs produced in the fireball near midrapidity. Clearly, these two contributions have very different dependence on beam energy, and disentangling them has a good potential to generate new insights. Towards this end, the STAR collaboration has defined [47] net-proton directed flow according to

$$
\left[v_{1}(y)\right]_{p}=r(y)\left[v_{1}(y)\right]_{\bar{p}}+[1-r(y)]\left[v_{1}(y)\right]_{\text {net- } p},
$$

where $r(y)$ is the observed rapidity dependence of the antiproton to proton ratio. Net-kaon $v_{1}(y)$ is defined analogously, with $K^{+}$and $K^{-}$substituted for $p$ and $\bar{p}$, respectively [90].

STAR's measurements of net-proton and net-kaon directed flow slope as a function of beam energy are reproduced in Figure 12 [90]. The net-proton directed flow shows a double sign change, and a clear minimum around the same beam energy where the proton directed flow has its minimum. This coincidence is not surprising, since 

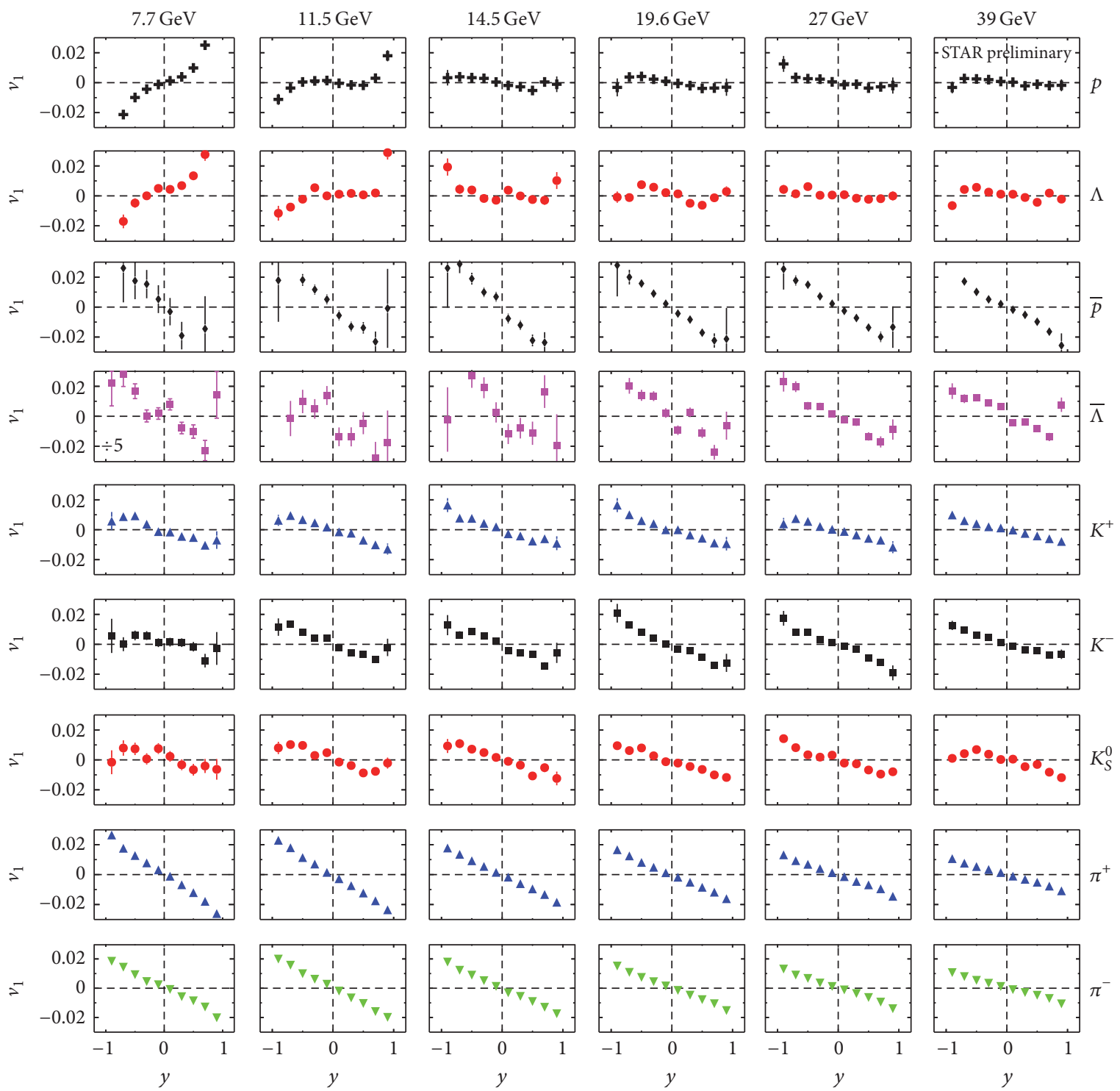

Figure 10: Directed flow as a function of rapidity for $p, \Lambda, \bar{p}, \bar{\Lambda}, K^{ \pm}, K_{S}^{0}$, and $\pi^{ \pm}$in $10-40 \%$ centrality Au+Au collisions, at $\sqrt{s_{\mathrm{NN}}}$ values of 7.7, $11.5,14.5,19.6,27$, and $39 \mathrm{GeV}[47,90]$. The magnitude of $v_{1}$ for $\bar{\Lambda}$ at $7.7 \mathrm{GeV}$ is divided by 5 to fit on the same vertical scale as all the other panels.

antibaryon production is low at and below the beam energy of the minimum, and therefore net-proton and proton observables only begin to deviate from each other at energies above the minimum.

The observed minimum in directed flow for protons and net protons resembles the predicted "softest point collapse" of directed flow $[19,22]$, and these authors are open to an interpretation in terms of a first-order phase transition. Other theorists (see Section 5) point out that mechanisms other than a first-order phase transition can cause a drop in pressure (a softening of the equation of state) and therefore a definitive conclusion requires more research.

The net-kaon directed flow reproduced in Figure 12 [90] shows close agreement with the net-proton result near and above $11.5 \mathrm{GeV}$ but deviates very strongly at $7.7 \mathrm{GeV}$. This deviation is not understood. To date, all of the several model comparisons with STAR $v_{1}$ measurements at BES energies have considered only particle $v_{1}$ as opposed to net-particle $v_{1}$.

\section{Recent Model Calculations of Directed Flow}

Models that explicitly incorporate properties of the Quantum Chromodynamics phase diagram and its equation of state (typically hydrodynamic models) suggest that the magnitude of directed flow is an excellent indicator of the relative pressure during the early, high-density stage of the collision. Therefore, directed flow at sub-AGS beam energies can reveal information about hadron gas incompressibility, while at the higher energies that are a focus of the present review, it can 


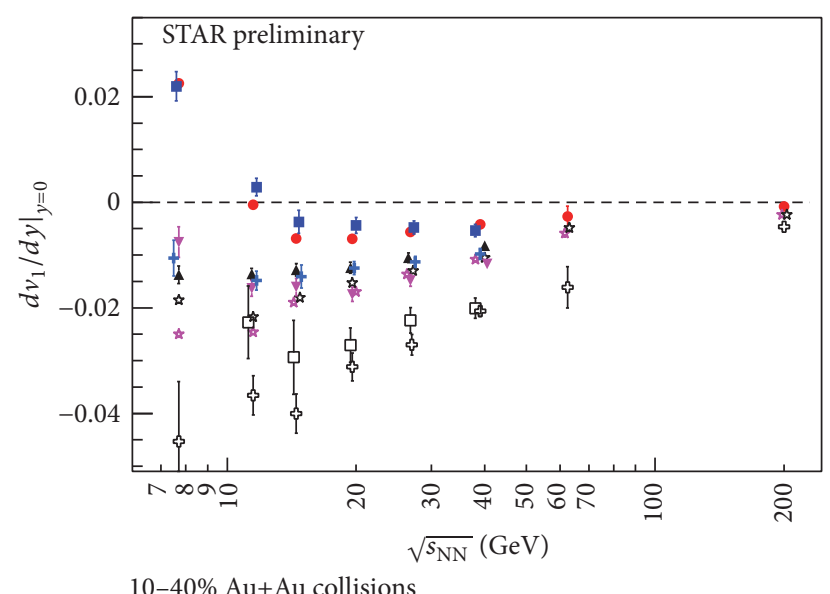

$10-40 \% \mathrm{Au}+\mathrm{Au}$ collisions

\begin{tabular}{|c|c|c|}
\hline - $p$ & $\Leftrightarrow \bar{p}$ & $\star \pi^{+}$ \\
\hline म $\pi^{-}$ & $\Delta K^{+}$ & - $\Lambda$ \\
\hline$\nabla K^{-}$ & $\square \bar{\Lambda}$ & $+K_{S}^{0}$ \\
\hline
\end{tabular}

FIGURE 11: Beam energy dependence of $v_{1}(y)$ slope for $p, \Lambda, \bar{p}, \bar{\Lambda}$, $K^{ \pm}, K_{S}^{0}$, and $\pi^{ \pm}$in $10-40 \%$ centrality Au+Au collisions $[47,90]$.

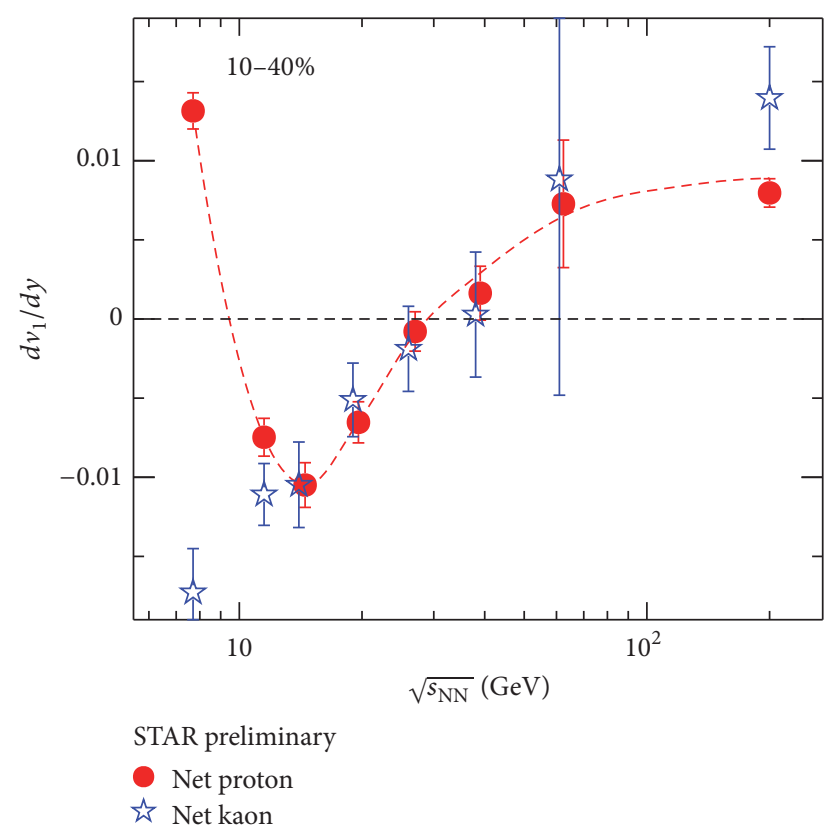

FIGURE 12: Beam energy dependence of the slope of $v_{1}(y)$ for net protons and net kaons in $10-40 \%$ centrality $\mathrm{Au}+\mathrm{Au}$ collisions, as reported by STAR $[47,90]$.

flag the softening, or drop in pressure, that may accompany a transition to a different phase, notably Quark Gluon Plasma. For example, there may be a spinodal decomposition associated with a first-order phase transition [92, 93], which would cause a large softening effect. However, interpretation of flow measurements is not straightforward, and it is known that directed flow can also be sensitive to poorly understood model inputs like momentum-dependent potentials [91] in the nuclear medium. More theoretical work is needed to elucidate the quantitative connection between softening signatures, like the beam energy dependence directed flow, and QCD phase changes.

During the period since publication of the STAR BES directed flow results in 2014, there have been several theoretical papers $[19,20,91,94,95]$ aimed towards interpretation of these measurements. The Frankfurt hybrid model [96] used for the data comparison by Steinheimer et al. [95] is based on a Boltzmann transport approach similar to UrQMD for the initial and late stages of the collision process, while a hydrodynamic evolution is employed for the intermediate hot and dense stage. The equation of state for the hydro stage includes crossover and first-order phase transition options. The data comparison by Konchakovski et al. [20] uses the Parton-Hadron String Dynamics (PHSD) model [72] of the Giessen group, a microscopic approach with a crossover equation of state having properties similar to the crossover of lattice QCD [87-89]. The PHSD code also has a mode named Hadron String Dynamics (HSD), which features purely hadronic physics throughout the collision evolution and which yields directed flow predictions in close agreement with those [47] of the UrQMD model. The data comparison by Ivanov and Soldatov [94] uses a relativistic 3-fluid hydrodynamic model (3FD) [97] with equations of state that include a crossover option and a first-order phase transition option. The most recent comparison to the STAR BES $v_{1}$ data, by Nara et al. [19], uses the Jet AA Microscopic (JAM) model [98]. JAM is a purely hadronic Boltzmann transport code, but the authors of [19] introduce an option to switch from the normal stochastic binary scattering style to a modified style where the elementary 2-body scatterings are always oriented like attractive orbits $[99,100]$. They argue that the switch-over from random to attractive binary orbits mimics the softening effect of a first-order phase transition.

Figure 13 focuses on the most promising directed flow measurement from the RHIC Beam Energy Scan, namely, the $d v_{1} /\left.d y\right|_{y \sim 0}$ for protons at $10-40 \%$ centrality, and summarizes recent model comparisons [19, 20, 94, 95] with these data. These authors are largely in agreement that the data disfavor models with purely hadronic physics. However, some conclude that a crossover deconfinement transition is favored $[20,94]$, while others conclude that a first-order phase transition is still a possible explanation [19]. Note that the argument of Nara et al. [19] is that a more sophisticated implementation of a first-order phase transition would transition from the "JAM" curve at low BES energies to the "JAM-attractive" curve at higher BES energies.

Overall, Figure 13 underlines the fact that no option in any of the model calculations to date reproduces, even qualitatively, the most striking feature of the data, namely, the minimum in proton directed flow in the region of $\sqrt{s_{\mathrm{NN}}} \sim$ $10-20 \mathrm{GeV}$. It is also noteworthy that the $v_{1}$ difference between nominally equivalent equations of state implementations in different models is very large. For example, the differences in $d v_{1} / d y$ between the 1st-order phase transition in the hybrid model [95] and a similar nominal quantity for the 3FD model [94] (see Figure 14) are currently more than an order of magnitude larger than the experimental measurement being interpreted and are larger still than the error on the measured data. 


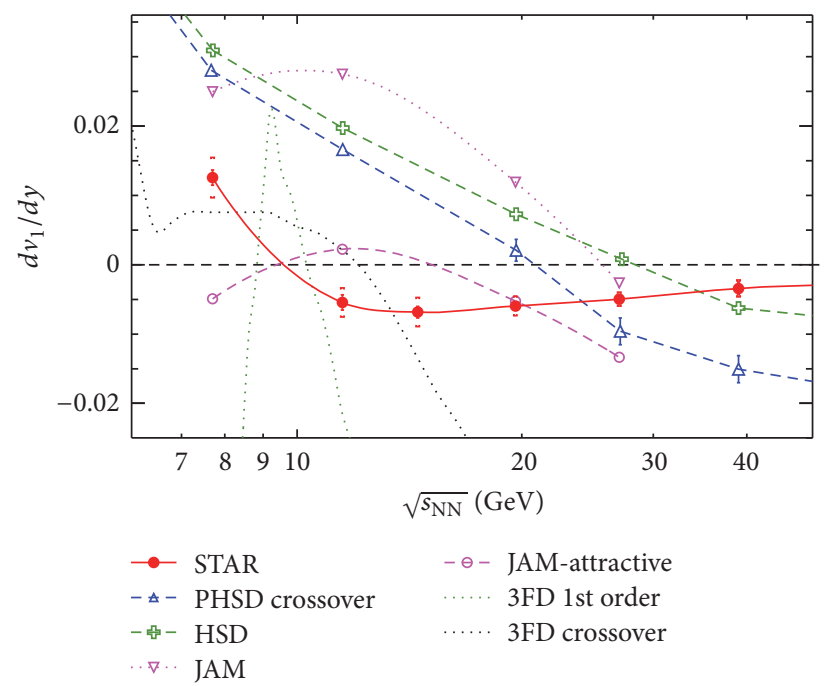

Figure 13: Beam energy dependence of directed flow slope for protons in $10-40 \%$ centrality $\mathrm{Au}+\mathrm{Au}$ from the STAR experiment, compared with recent available model calculations [19, 20, 94]. All the experimental data are from [47] except for one energy point, $\sqrt{s_{\mathrm{NN}}}=14.5 \mathrm{GeV}$ [90], which should be considered a preliminary measurement. The Frankfurt hybrid model [95] and a pure hydro calculation with particle freeze-out at constant energy density [95] both lie above the data and are off-scale at all BES energies.

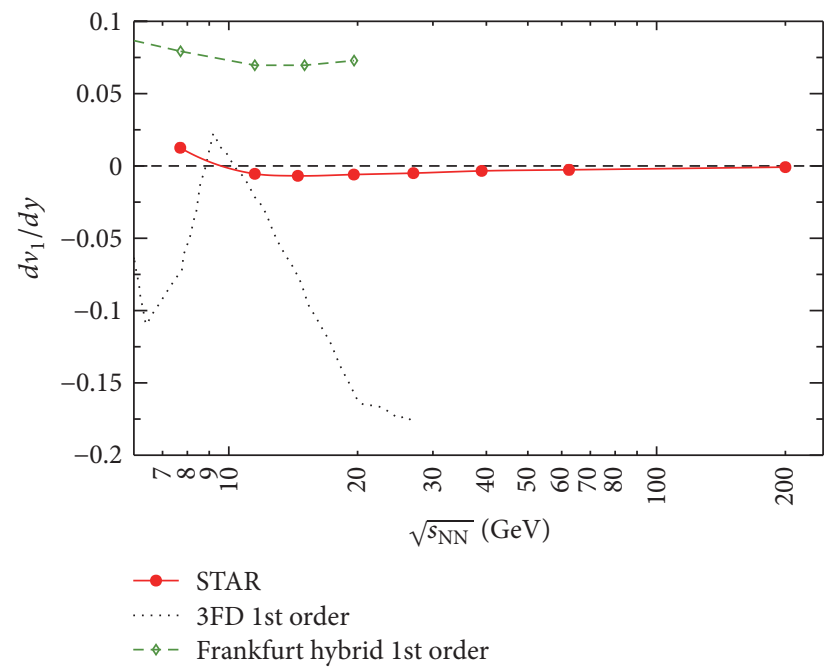

Figure 14: Beam energy dependence of directed flow slope for protons in $10-40 \%$ centrality $\mathrm{Au}+\mathrm{Au}$ from the STAR experiment, compared with recent hybrid [95] and 3FD [94] model calculations. All the experimental data are from [47] except for one energy point, $\sqrt{s_{\mathrm{NN}}}=14.5 \mathrm{GeV}$ [90], which should be considered a preliminary measurement.

\section{Summary and Outlook}

In this review, we discuss heavy-ion directed flow results for charged particles and for identified particle types, covering beam energies from the Brookhaven AGS to the CERN LHC. Charged particle directed flow measurements have been published as a function of transverse momentum, pseudorapidity, and collision centrality, while $\mathrm{Cu}+\mathrm{Cu}$ and $\mathrm{Au}+\mathrm{Au}$ have also been compared. The charged particle directed flow magnitude at the LHC is a factor of three smaller than that at top RHIC energy. The observations from RHIC suggest that the charged particle directed flow is independent of system size but depend on the incident beam energy. Limiting fragmentation scaling is observed for $v_{1}$ at RHIC energies, but entropy-driven multiplicity scaling in terms of $d N_{\text {ch }} / d \eta$ is not seen at RHIC. In mass-asymmetric collisions, specifically $\mathrm{Cu}+\mathrm{Au}$, recent directed flow measurements at $\sqrt{s_{\mathrm{NN}}}=200 \mathrm{GeV}$ have opened a new window into quark and antiquark formation at the very earliest times of the collision evolution $(t \leq 0.25 \mathrm{fm} / c)$ and could clarify theoretical and experimental questions related to the Chiral Magnetic Effect and the Chiral Magnetic Wave.

Measurements of $v_{1}$ for identified species offer deeper insights into the development of hydrodynamic flow. Opposite $v_{1}$ for pions and protons at AGS/SPS and at lower RHIC energies suggests an important role for nuclear shadowing. Signals of antiflow of neutral kaons in AGS/E895 together with kaon measurements in the RHIC Beam Energy Scan region point to kaon-nucleon potential effects. The single sign change in proton $v_{1}$ slope and a double sign change in netproton $v_{1}$ slope, with a clear minimum around $\sqrt{s_{\mathrm{NN}}} \sim 11.5-$ $19.6 \mathrm{GeV}$, show a qualitative resemblance to a hydrodynamic model prediction called "softest point collapse of flow." This original prediction assumed a first-order phase transition, but a crossover from hadron gas to a deconfined phase can also cause softening (a drop in pressure). None of the current state-of-the-art models can explain the main features of the STAR directed flow measurements, and different models with nominally similar equations of state diverge from each other very widely over the BES range.

Looking ahead to likely developments during the period 2017-2018 in the area of directed flow at $\sqrt{s_{\mathrm{NN}}}$ of a few $\mathrm{GeV}$ and above, we can expect new BES Phase-I results for the $\phi$ meson, as well as final publication of current preliminary RHIC Beam Energy Scan Phase-I results, like those in [90]. We can also expect parallel theoretical work on related physics and interpretation of the newest data. The preliminary results for new particle species like $\Lambda$ s and charged and neutral kaons, as well as BES $v_{1}$ for protons, $\Lambda$, and pions in narrow bins of centrality (nine bins' spanning $0-80 \%$ centrality) amount to very stringent constraints on the next round of theoretical interpretation in terms of the QCD phase diagram and equation of state.

More comprehensive measurements of the many phenomenological aspects of directed flow outlined in this review will be possible beginning in the year 2019, as a consequence of the much increased statistics of Phase-II of the RHIC Beam Energy Scan (to take data in 2019 and 2020), in conjunction with the anticipated upgrades to the performance of the STAR detector [101]. Thereafter, new facilities like FAIR [102] in Germany, NICA [103] in Russia, and J-PARC-HI [104, 105] in Japan, will begin coming online. These new dedicated facilities will further strengthen worldwide research on the QCD phase diagram at high baryon chemical potential. 


\section{Competing Interests}

The authors declare that there is no conflict of interests regarding the publication of this paper.

\section{Acknowledgments}

This work was supported in part by the Office of Nuclear Physics within the US DOE Office of Science, under Grant DE-FG02-89ER40531. The authors are grateful to the following colleagues for insightful advice and discussions: J. Auvinen, H. Caines, D. Cebra, V. Dexheimer, M. Lisa, W. Llope, G. Odyniec, Y. Pandit, H. Petersen, A. Poskanzer, H. G. Ritter, J. Steinheimer, H. Stöcker, A. H. Tang, S. Voloshin, G. Wang, N. $\mathrm{Xu}$, and $\mathrm{Z}$. Xu.

\section{References}

[1] I. Arsene, I. G. Bearden, D. Beavis et al., "Quark-gluon plasma and color glass condensate at RHIC? The perspective from the BRAHMS experiment," Nuclear Physics A, vol. 757, no. 1-2, pp. 1-27, 2005.

[2] B. B. Back, M. D. Baker, M. Ballintijn et al., "The PHOBOS perspective on discoveries at RHIC," Nuclear Physics A, vol. 757, no. 1-2, pp. 28-101, 2005.

[3] J. Adams, M. M. Aggarwal, Z. Ahammed et al., "Experimental and theoretical challenges in the search for the quark-gluon plasma: the STAR Collaboration's critical assessment of the evidence from RHIC collisions," Nuclear Physics A, vol. 757, no. 1-2, pp. 102-183, 2005.

[4] K. Adcox, S. S. Adler, S. Afanasiev et al., "Formation of dense partonic matter in relativistic nucleus-nucleus collisions at RHIC: experimental evaluation by the PHENIX Collaboration," Nuclear Physics A, vol. 757, no. 1-2, pp. 184-283, 2005.

[5] B. Müller and J. L. Nagle, "Results from the relativistic heavy ion collider," Annual Review of Nuclear and Particle Science, vol. 56, no. 1, pp. 93-135, 2006.

[6] B. Jacak and P. Steinberg, "Creating the perfect liquid in heavyion collisions," Physics Today, vol. 63, no. 5, pp. 39-43, 2010.

[7] S. A. Bass, H. Caines, B. A. Cole et al., Hot \& Dense QCD Matter, White Paper Submitted to the 2012 Nuclear Science Advisory Committee, https://www.bnl.gov/npp/docs/Bass_RHI_WP_final .pdf.

[8] W. Reisdorf and H. G. Ritter, "Collective flow in heavy-ion collisions," Annual Review of Nuclear and Particle Science, vol. 47, pp. 663-709, 1997.

[9] H. Sorge, "Elliptical flow: a signature for early pressure in ultrarelativistic nucleus-nucleus collisions," Physical Review Letters, vol. 78, no. 12, pp. 2309-2312, 1997.

[10] N. Herrmann, J. P. Wessels, and T. Wienold, "Collective flow in heavy-ion collisions," Annual Review of Nuclear and Particle Science, vol. 49, no. 1, pp. 581-632, 1999.

[11] M. Gyulassy, K. A. Frankel, and H. Stöcker, "Do nuclei flow at high energies?” Physics Letters B, vol. 110, no. 3-4, pp. 185-188, 1982.

[12] P. Danielewicz and M. Gyulassy, "Jacobian free global event analysis," Physics Letters B, vol. 129, no. 5, pp. 283-288, 1983.

[13] H. A. Gustafsson, H. H. Gutbrod, B. Kolb et al., "Collective flow observed in relativistic nuclear collisions," Physical Review Letters, vol. 52, no. 18, p. 1590, 1984.
[14] S. Voloshin and Y. Zhang, "Flow study in relativistic nuclear collisions by Fourier expansion of azimuthal particle distributions," Zeitschrift fur Physik C-Particles and Fields, vol. 70, no. 4, pp. 665-671, 1996.

[15] A. M. Poskanzer and S. A. Voloshin, "Methods for analyzing anisotropic flow in relativistic nuclear collisions," Physical Review C-Nuclear Physics, vol. 58, no. 3, pp. 1671-1678, 1998.

[16] A. Bilandzic, R. Snellings, and S. Voloshin, "Flow analysis with cumulants: direct calculations," Physical Review C-Nuclear Physics, vol. 83, no. 4, Article ID 044913, 2011.

[17] D. Teaney and L. Yan, "Triangularity and dipole asymmetry in relativistic heavy ion collisions," Physical Review C, vol. 83, no. 6, Article ID 064904, 2011.

[18] M. Luzum and J.-Y. Ollitrault, "Directed flow at midrapidity in heavy-ion collisions," Physical Review Letters, vol. 106, no. 10, Article ID 102301, 4 pages, 2011.

[19] Y. Nara, A. Ohnishi, and H. Stöcker, "Examination of directed flow as a signature of the softest point of the equation of state in QCD matter," Physical Review C, vol. 94, no. 3, Article ID 034906, 2016.

[20] V. P. Konchakovski, W. Cassing, Y. B. Ivanov, and V. D. Toneev, "Examination of the directed flow puzzle in heavy-ion collisions," Physical Review C-Nuclear Physics, vol. 90, no. 1, Article ID 014903, 2014.

[21] U. Heinz, "Early collective expansion: relativistic hydrodynamics and the transport properties of QCD matter," in Relativistic Heavy Ion Physics, R. Stock, Ed., vol. 23 of Landolt-BörnsteinGroup I Elementary Particles, Nuclei and Atoms, chapter 5, pp. 240-292, Springer, New York, NY, USA, 2010.

[22] H. Stöcker, "Collective flow signals the quark-gluon plasma," Nuclear Physics A, vol. 750, no. 1, pp. 121-147, 2005.

[23] S. A. Bass, M. Belkacem, M. Bleicher et al., "Microscopic models for ultrarelativistic heavy ion collisions," Progress in Particle and Nuclear Physics, vol. 41, pp. 255-369, 1998.

[24] M. Bleicher, E. Zabrodin, C. Spieles et al., "Relativistic hadronhadron collisions in the ultra-relativistic quantum molecular dynamics model," Journal of Physics G: Nuclear and Particle Physics, vol. 25, no. 9, pp. 1859-1896, 1999.

[25] S. Z. Belenkij and L. D. Landau, "Hydrodynamic theory of multiple production of particles," Il Nuovo Cimento, vol. 3, no. 1, pp. 15-31, 1956.

[26] G. F. Chapline, M. H. Johnson, E. Teller, and M. S. Weiss, "Highly excited nuclear matter," Physical Review D, vol. 8, no. 12, pp. 4302-4308, 1973.

[27] W. Scheid, H. Müller, and W. Greiner, "Nuclear shock waves in heavy-ion collisions," Physical Review Letters, vol. 32, no. 13, pp. 741-745, 1974.

[28] J. Hofmann, H. Stöcker, U. Heinz, W. Scheid, and W. Greiner, "Possibility of detecting density isomers in high-density nuclear mach shock waves," Physical Review Letters, vol. 36, no. 2, pp. 88-91, 1976.

[29] H. Stöcker, J. A. Maruhn, and W. Greiner, "Collective sideward flow of nuclear matter in violent high-energy heavy-ion collisions," Physical Review Letters, vol. 44, no. 11, pp. 725-728, 1980.

[30] J. Barrette, J. Barrette, R. Bellwied et al., "Directed flow and particle production in $\mathrm{Au}+\mathrm{Au}$ collisions from experiment E877 at the AGS," Nuclear Physics A, vol. 590, no. 1-2, pp. 259-269, 1995. 
[31] J. Barrette, R. Bellwied, S. Bennett et al., "Proton and pion production relative to the reaction plane in $\mathrm{Au}+\mathrm{Au}$ collisions at $11 A \mathrm{GeV} /$ c," Physical Review C, vol. 56, no. 6, pp. 3254-3264, 1997.

[32] J. Barrette, R. Bellwied, S. Bennett et al., "Directed flow of light nuclei in $\mathrm{Au}+\mathrm{Au}$ collisions at $10.8 \mathrm{~A} \mathrm{GeV} / c$," Physical Review $C$, vol. 59, no. 2, pp. 884-888, 1999.

[33] J. Barrette, R. Bellwied, S. Bennett et al., "Directed flow of antiprotons in Au+Au collisions at AGS," Physics Letters B, vol. 485, no. 4, pp. 319-326, 2000.

[34] H. Liu, N. N. Ajitanand, J. Alexander et al., "Sideward flow in $\mathrm{Au}+\mathrm{Au}$ Collisions between $2 \mathrm{~A}$ and $8 \mathrm{~A} \mathrm{GeV,"} \mathrm{Physical} \mathrm{Review}$ Letters, vol. 84, no. 24, p. 5488, 2000.

[35] P. Chung, N. N. Ajitanand, J. M. Alexander et al., "Antiflow of $K_{s}^{0}$ mesons in $6 \mathrm{~A} \mathrm{GeV} \mathrm{Au+Au} \mathrm{collisions,"} \mathrm{Physical} \mathrm{Review} \mathrm{Letters,}$ vol. 85 , no. 5, pp. 940-943, 2000.

[36] P. Chung, N. N. Ajitanand, J. M. Alexander et al., "Directed flow of $\Lambda$ hyperons in (2-6 )A GeV Au $+A u$ collisions," Physical Review Letters, vol. 86, no. 12, pp. 2533-2536, 2001.

[37] C. Pinkenburg and E895 Collaboration, "Production and collective behavior of strange particles in $\mathrm{Au}+\mathrm{Au}$ collisions at 28 A GeV," Nuclear Physics A, vol. 698, no. 1-4, pp. 495-498, 2002.

[38] C. Pinkenburg, "Production and collective behavior of strange particles in $\mathrm{Au}+\mathrm{Au}$ collisions at 2-8 A GeV," Nuclear Physics A, vol. 698, no. 1-4, pp. 495-498, 2002.

[39] H. Appelshäuser, J. Bächler, S. J. Bailey et al., "Directed and elliptic flow in $158 \mathrm{GeV} /$ nucleon $\mathrm{Pb}+\mathrm{Pb}$ collisions," Physical Review Letters, vol. 80, no. 19, p. 4136, 1998.

[40] C. Alt, T. Anticic, B. Baatar et al., "Directed and elliptic flow of charged pions and protons in $\mathrm{Pb}+\mathrm{Pb}$ collisions at $40 \mathrm{~A}$ and 158 A GeV," Physical Review C, vol. 68, no. 3, Article ID 034903, 2003.

[41] J. Adams, M. M. Aggarwal, Z. Ahammed et al., "Azimuthal anisotropy in $\mathrm{Au}+\mathrm{Au}$ collisions at $\sqrt{s_{\mathrm{NN}}}=200 \mathrm{GeV}$," Physical Review C, vol. 72, no. 1, Article ID 014904, 23 pages, 2005.

[42] B. B. Back, M. D. Baker, M. Ballintijn et al., "Energy dependence of directed flow over a wide range of pseudorapidity in $\mathrm{Au}+\mathrm{Au}$ Collisions at the BNL relativistic heavy ion collider," Physical Review Letters, vol. 97, Article ID 012301, 2006.

[43] J. Adams, M. M. Aggarwal, Z. Ahammed, and X. Zuo, "Directed flow in $\mathrm{Au}+\mathrm{Au}$ collisions at $\sqrt{s_{N N}}=62.4 \mathrm{GeV}$," Physical Review C, vol. 73, no. 3, Article ID 034903, 2006.

[44] B. I. Abelev, M. M. Aggarwal, Z. Ahammed et al., "System-size independence of directed flow measured at the BNL relativistic heavy-ion collider," Physical Review Letters, vol. 101, no. 25, Article ID 252301, 2008.

[45] G. Agakishiev, M. M. Aggarwal, Z. Ahammed et al., "Directed and elliptic flow of charged particles in $\mathrm{Cu}+\mathrm{Cu}$ collisions at $\sqrt{s_{N N}}=22.4$ Gev," Physical Review C, vol. 85 , no. 1 , Article ID 014901, 2012.

[46] L. Adamczyk, G. Agakishiev, M. M. Aggarwal et al., "Directed flow of identified particles in $\mathrm{Au}+\mathrm{Au}$ collisions at $\sqrt{\boldsymbol{s}_{N N}}=$ $200 \mathrm{GeV}$ at RHIC," Physical Review Letters, vol. 108, Article ID 202301, 2012.

[47] L. Adamczyk, J. K. Adkins, G. Agakishiev et al., "Beam-energy dependence of the directed flow of protons, antiprotons, and pions in Au+Au collisions," Physical Review Letters, vol. 112, Article ID 162301, 2014.

[48] L. P. Csernai and D. Röhrich, "Third flow component as QGP signal," Physics Letters B, vol. 458, no. 4, pp. 454-459, 1999.
[49] J. Brachmann, S. Soff, A. Dumitru et al., "Antiflow of nucleons at the softest point of the equation of state," Physical Review C, vol. 61, no. 2, Article ID 024909, 2000.

[50] R. J. M. Snellings, H. Sorge, S. A. Voloshin, F. Q. Wang, and N. $\mathrm{Xu}$, "Novel rapidity dependence of directed flow in high-energy heavy-ion collisions," Physical Review Letters, vol. 84, no. 13, pp. 2803-2805, 2000.

[51] Y. Guo, F. Liu, and A. Tang, "Directed flow of transported and nontransported protons in $\mathrm{Au}+\mathrm{Au}$ collisions from an ultrarelativistic quantum molecular dynamics model," Physical Review C, vol. 86, no. 4, Article ID 044901, 4 pages, 2012.

[52] D. Kovalev, H. Heckler, M. Ben-Chorin, G. Polisski, M. Schwartzkopff, and F. Koch, "Breakdown of the $k$-conservation rule in Si nanocrystals," Physical Review Letters, vol. 81, no. 13, pp. 2803-2806, 1998.

[53] B. Abelev, J. Adam, D. Adamovà et al., "Directed flow of charged particles at midrapidity relative to the spectator plane in $\mathrm{Pb}-\mathrm{Pb}$ collisions at $\sqrt{s_{\mathrm{NN}}}=2.76 \mathrm{TeV}$," Physical Review Letters, vol. 111, no. 23, Article ID 232302, 11 pages, 2013.

[54] STAR ZDC-SMD Proposal, STAR Note SN-0448, 2003, https://drupal.star.bnl.gov/STAR/starnotes/public/sn0448.

[55] G. Wang, Correlations relative to the reaction plane at the relativistic heavy ion collider based on transverse deflection of spectator neutrons [Ph.D. thesis], Kent State University, 2005, https://drupal.star.bnl.gov/STAR/theses/ph-d/gang-wang.

[56] C. E. Allgower, B. D. Anderson, A. R. Baldwin et al., "The STAR endcap electromagnetic calorimeter," Nuclear Instruments and Methods in Physics Research Section A: Accelerators, Spectrometers, Detectors and Associated Equipment, vol. 499, no. 2-3, pp. 740-750, 2003.

[57] U. Heinz and P. F. Kolb, "Rapidity dependent momentum anisotropy at RHIC," Journal of Physics G: Nuclear and Particle Physics, vol. 30, no. 8, pp. S1229-S1233, 2004.

[58] B. I. Abelevi, M. M. Aggarwalad, Z. Ahammed et al., "Energy dependence of $\pi^{ \pm}, p$ and $\bar{p}$ transverse momentum spectra for $\mathrm{Au}+\mathrm{Au}$ collisions at $\sqrt{s_{N N}}=62.4$ and $200 \mathrm{GeV}$," Physics Letters $B$, vol. 655, no. 3-4, pp. 104-113, 2007.

[59] J. Bleibel, G. Burau, and C. Fuchs, "Anisotropic flow in $\mathrm{Pb}$ $+\mathrm{Pb}$ collisions at LHC from the quark-gluon string model with parton rearrangement," Physics Letters, Section B: Nuclear, Elementary Particle and High-Energy Physics, vol. 659, no. 3, pp. 520-524, 2008.

[60] L. P. Csernai, V. K. Magas, H. Stöcker, and D. D. Strottman, "Fluid dynamical prediction of changed vl flow at energies available at the CERN Large Hadron Collider," Physical Review C-Nuclear Physics, vol. 84, no. 2, Article ID 024914, 2011.

[61] Z.-W. Lin and C. M. Ko, "Partonic effects on the elliptic flow at relativistic heavy ion collisions," Physical Review C, vol. 65, no. 3, Article ID 034904, 2002.

[62] Z.-W. Lin, C. M. Ko, B.-A. Li, B. Zhang, and S. Pal, "Multiphase transport model for relativistic heavy ion collisions," Physical Review C, vol. 72, no. 6, Article ID 064901, 2005.

[63] L.-W. Chen, V. Greco, C. M. Ko, and P. F. Kolb, "Pseudorapidity dependence of anisotropic flows in relativistic heavy-ion collisions," Physics Letters B, vol. 605, no. 1-2, pp. 95-100, 2005.

[64] R. P. Feynman, "Very high-energy collisions of hadrons," Physical Review Letters, vol. 23, no. 24, pp. 1415-1417, 1969.

[65] J. Benecke, T. T. Chou, C.-N. Yang, and E. Yen, "Hypothesis of limiting fragmentation in high-energy collisions," Physical Review, vol. 188, no. 5, pp. 2159-2169, 1969. 
[66] A. Adare, C. Aidala, N. N. Ajitanand et al., "Measurements of directed, elliptic, and triangular flow in $\mathrm{Cu}+\mathrm{Au}$ collisions at $\sqrt{s_{\mathrm{NN}}}=200 \mathrm{GeV}$," https://arxiv.org/abs/1509.07784.

[67] H. Caines, "Is soft physics entropy driven?" The European Physical Journal C, vol. 49, no. 1, pp. 297-301, 2007.

[68] M. Lisa, "Femtoscopy in heavy ion collisions: wherefore, whence, and whither?" in Proceedings of the Multiparticle Dynamics: 35th International Symposium on Multiparticle Dynamics; and Workshop on Particle Correlations and Femtoscopy, vol. 828 of AIP Conference Proceedings, p. 226, Kromeriz, Czech Republic, August 2005.

[69] B. Alver, B. B. Back, M. D. Baker et al., "System size, energy, pseudorapidity, and centrality dependence of elliptic flow," Physical Review Letters, vol. 98, Article ID 242302, 2007.

[70] L. Adamczyk, J. K. Adkins, G. Agakishiev et al., "Chargedependent directed flow in $\mathrm{Cu}+\mathrm{Au}$ collisions at $\sqrt{s_{N N}}=$ $200 \mathrm{GeV}$," https://arxiv.org/abs/1608.04100.

[71] M. D. Partlan, S. Albergo, F. Bieser et al., "Fragment flow in $\mathrm{Au}+\mathrm{Au}$ collisions," Physical Review Letters, vol. 75, no. 11, pp. 2100-2103, 1995.

[72] W. Cassing, "From Kadanoff-Baym dynamics to off-shell parton transport," The European Physical Journal Special Topics, vol. 168, no. 1, pp. 3-87, 2009.

[73] V. Voronyuk, V. D. Toneev, S. A. Voloshin, and W. Cassing, "Charge-dependent directed flow in asymmetric nuclear collisions," Physical Review C, vol. 90, no. 6, Article ID 064903, 2014.

[74] https://www.desy.de/hlzeus/combined_results/herapdftable/.

[75] D. Kharzeev, R. D. Pisarski, and M. H. G. Tytgat, "Possibility of spontaneous parity violation in hot QCD," Physical Review Letters, vol. 81, no. 3, pp. 512-515, 1998.

[76] D. Kharzeev and R. D. Pisarski, "Pionic measures of parity and CP violation in high energy nuclear collisions," Physical Review D, vol. 61, no. 11, Article ID 111901, 2000.

[77] D. E. Kharzeev and H. Yee, "Chiral magnetic wave," Physical Review D, vol. 83, no. 8, Article ID 085007, 2011.

[78] Y. Burnier, D. E. Kharzeev, J. Liao, and H.-U. Yee, "Chiral magnetic wave at finite baryon density and the electric quadrupole moment of the quark-gluon plasma," Physical Review Letters, vol. 107, no. 5, Article ID 052303, 2011.

[79] S. A. Voloshin, "Transverse radial expansion and directed flow," Physical Review C-Nuclear Physics, vol. 55, no. 4, pp. R1630R1632, 1997.

[80] P. Kolb and U. Heinz, "Hydrodynamic description of ultrarelativistic heavy-ion collisions," https://arxiv.org/abs/nucl-th/ 0305084.

[81] P. Huovinen and P. Ruuskanen, "Hydrodynamic models for heavy ion collisions," Annual Review of Nuclear and Particle Science, vol. 56, no. 1, pp. 163-206, 2006.

[82] N. Borghini, P. M. Dinh, and J. Ollitrault, "Analysis of directed flow from elliptic flow," Physical Review C, vol. 66, no. 1, Article ID 014905, 2002.

[83] P. Danielewicz and G. Odyniec, "Transverse momentum analysis of collective motion in relativistic nuclear collisions," Physics Letters B, vol. 157, no. 2-3, pp. 146-150, 1985.

[84] M. Isse, A. Ohnishi, N. Otuka, P. K. Sahu, and Y. Nara, "Mean-field effects on collective flow in high-energy heavy-ion collisions at 2-158A GeV energies," Physical Review C, vol. 72, no. 6, Article ID 064908, 2005.
[85] B. I. Abelev et al., "SN0493: experimental study of the QCD phase diagram \& search for the critical point: selected arguments for the Run-10 beam energy scan," (STAR collaboration), STAR Note SN0493, 2009, https://drupal.star.bnl.gov/STAR/ starnotes/public/sn0493.

[86] M. M. Aggarwal, Z. Ahammed, A. V. Alakhverdyants et al., "An experimental exploration of the QCD phase diagram: the search for the critical point and the onset of de-confinement," https://arxiv.org/abs/1007.2613.

[87] F. Karsch, C. R. Allton, S. Ejiri et al., "Where is the chiral critical point in 3-flavor QCD?" Nuclear Physics B-Proceedings Supplements, vol. 129-130, pp. 614-616, 2004.

[88] Y. Aoki, G. Endrodi, Z. Fodor, S. D. Katz, and K. K. Szabó, “The order of the quantum chromodynamics transition predicted by the standard model of particle physics," Nature, vol. 443, no. 7112, pp. 675-678, 2006.

[89] M. Cheng, P. Hegde, C. Jung et al., "Baryon number, strangeness, and electric charge fluctuations in QCD at high temperature," Physical Review D, vol. 79, no. 7, Article ID 074505, 12 pages, 2009.

[90] P. Shanmuganathan, "Beam-energy and centrality dependence of directed flow of identified particles," https://arxiv.org/abs/ 1512.09009 .

[91] W. Cassing, V. P. Konchakovski, A. Palmese, V. D. Toneev, and E. L. Bratkovskaya, "Parton/hadron dynamics in heavy-ion collisions at FAIR energies," EPJ Web of Conferences, vol. 95, Article ID 01004, 2015.

[92] P. Shukla and A. K. Mohanty, "Nucleation versus spinodal decomposition in a first order quark hadron phase transition," Physical Review C, vol. 64, no. 5, Article ID 054910, 2001.

[93] A. Bessa, E. S. Fraga, and B. W. Mintz, "Phase conversion in a weakly first-order quark-hadron transition," Physical Review D, vol. 79, no. 3, Article ID 034012, 2009.

[94] Yu. B. Ivanov and A. A. Soldatov, "Directed flow indicates a cross-over deconfinement transition in relativistic nuclear collisions," Physical Review C, vol. 91, no. 2, Article ID 024915, 9 pages, 2015.

[95] J. Steinheimer, J. Auvinen, H. Petersen, M. Bleicher, and H. Stöcker, "Examination of directed flow as a signal for a phase transition in relativistic nuclear collisions," Physical Review CNuclear Physics, vol. 89, no. 5, Article ID 054913, 2014.

[96] H. Petersen, J. Steinheimer, G. Burau, M. Bleicher, and H. Stöcker, "Fully integrated transport approach to heavy ion reactions with an intermediate hydrodynamic stage," Physical Review C-Nuclear Physics, vol. 78, no. 4, Article ID 044901, 2008.

[97] Y. B. Ivanov, V. N. Russkikh, and V. D. Toneev, "Relativistic heavy-ion collisions within three-fluid hydrodynamics: hadronic scenario," Physical Review C, vol. 73, Article ID 044904, 2006.

[98] Y. Nara, N. Otuka, A. Ohnishi, K. Niita, and S. Chiba, "Relativistic nuclear collisions at $10 \mathrm{~A} \mathrm{GeV}$ energies from $\mathrm{p}+\mathrm{Be}$ to $\mathrm{Au}+\mathrm{Au}$ with the hadronic cascade model," Physical Review C-Nuclear Physics, vol. 61, no. 2, article 024901, 2000.

[99] D. E. Kahana, D. Keane, Y. Pang, T. Schlagel, and S. Wang, "Collective flow from the intranuclear cascade model," Physical Review Letters, vol. 74, no. 22, pp. 4404-4407, 1995.

[100] D. E. Kahana, Y. Pang, and E. Shuryak, "Flow at Brookhaven AGS energy (11.6 GeV/nucleon): a barometer for high density effects?” Physical Review C, vol. 56, no. 1, pp. 481-485, 1997. 
[101] STAR collaboration, BES Phase-II Whitepaper, STAR Note SN0598, 2014, https://drupal.star.bnl.gov/STAR/starnotes/public/sn0598.

[102] http://www.fair-center.eu.

[103] http://nica.jinr.ru/tech.html.

[104] H. Sako, T. Chujo, T. Gunji et al., "Towards the heavy-ion program at J-PARC," Nuclear Physics A, vol. 931, pp. 1158-1162, 2014.

[105] H. Sako, T. Sakaguchi, M. Kaneta et al., White Paper for J-PARC Heavy-Ion Program, April 2016, http://silver.j-parc.jp/sako/ white-paper-v1.17.pdf. 

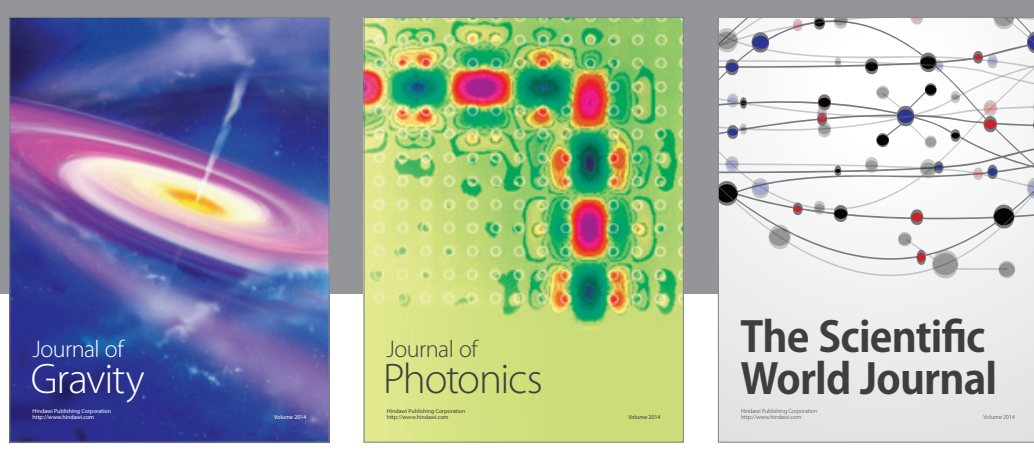

The Scientific World Journal
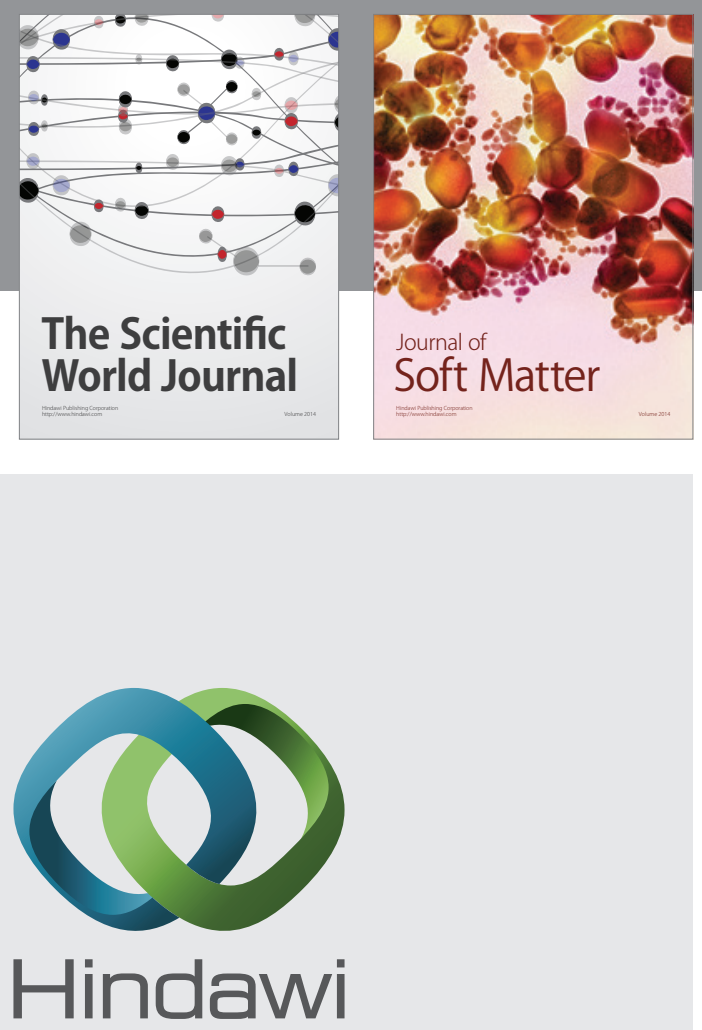

Submit your manuscripts at

http://www.hindawi.com

nternational Journal of

Statistical Mechanics
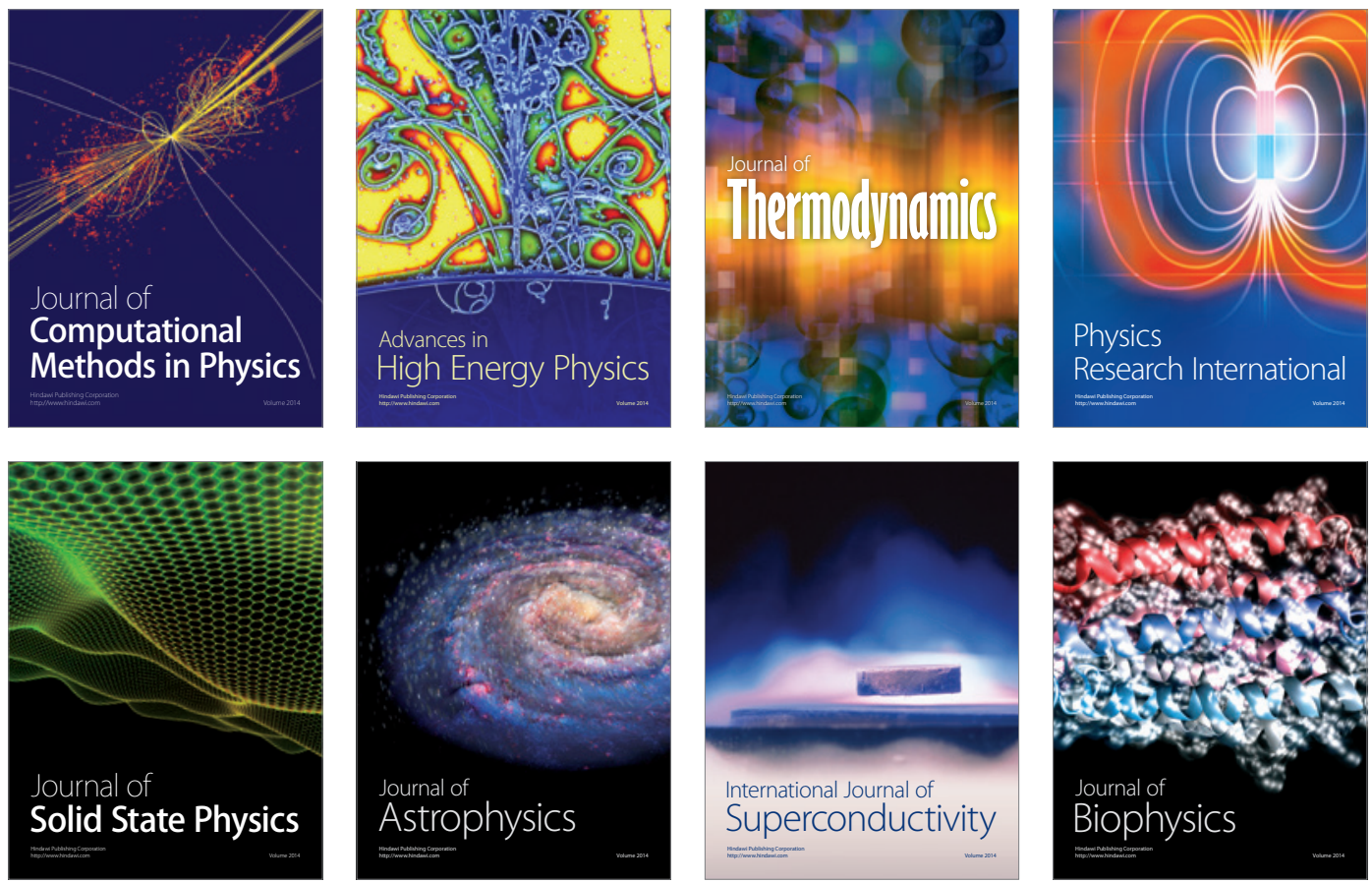
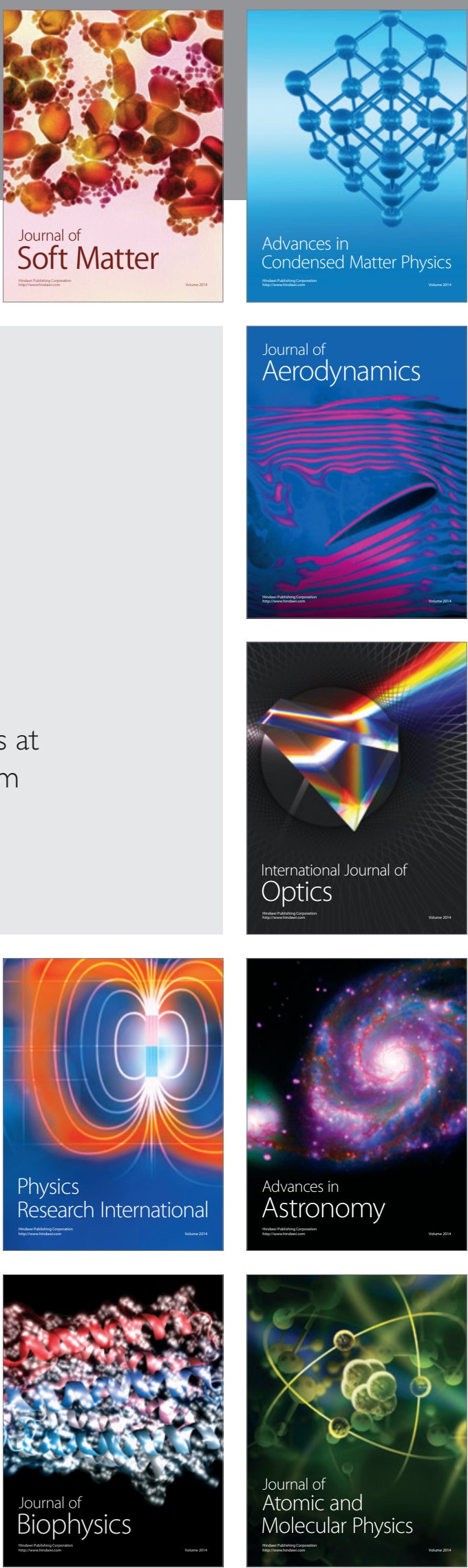